\title{
Electroweak effects in polarized muon-electron scattering
}

\author{
A. B. Arbuzov $\odot^{*}$ and S. G. Bondarenko® $\oplus^{\dagger}$ \\ Bogoliubov Laboratory of Theoretical Physics, Joint Institute for Nuclear Research, \\ Dubna 141980, Russia \\ L. V. Kalinovskaya๑, L. A. Rumyantsev@, and V. L. Yermolchyk $\oplus^{\ddagger}$ \\ Dzhelepov Laboratory of Nuclear Problems, Joint Institute for Nuclear Research, Dubna 141980, Russia
}

(Received 20 December 2021; accepted 2 February 2022; published 25 February 2022)

\begin{abstract}
A theoretical description of elastic polarized muon-electron scattering is presented. Complete one-loop electroweak radiative corrections are calculated with taking into account the exact dependence on the muon mass. The effects due to some higher-order corrections and the electroweak scheme dependence are analyzed. The case of longitudinally polarized fermions in the initial state is investigated. Analytical results are derived with the help of the SANC system. Numerical results are presented for unpolarized and polarized cross sections. Calculations are realized in the Monte Carlo integrator MCSANCee and generator ReneSANCe, which allow the implementation of any experimental cuts used in the analysis of elastic $\mu-e$ scattering data.
\end{abstract}

DOI: 10.1103/PhysRevD.105.033009

\section{INTRODUCTION}

Muon-electron scattering is one of the most pure QED processes of elementary particle interactions. So its cross section can be calculated perturbatively within the Standard Model with a very high precision. On the other hand, this process is suited for high-precision experimental measurements since it has a clear detector signature. This allows us to use this process for studies of electroweak (EW) and strong interaction effects, given the QED part is fully understood.

Radiative corrections in polarized electron muon elastic scattering were considered by [1] and later fully unpolarized but massive case at nest-to-leading order (NLO) was examined by [2].

The scattering of muons off polarized electrons was measured by SMC Collaboration at CERN in [3]. At the request of the SMC Collaboration, the working tool for estimating theoretical uncertainty under experimental conditions was our code $\mu$ ela [4]. The code for elastic polarized $\mu-e$ scattering was implemented at the QED level with the

\footnotetext{
*Also at Department of higher mathematics, Dubna State University, Dubna 141982, Russia.

bondarenko@jinr.ru

${ }^{*}$ Also at Institute for Nuclear Problems, Belarusian State University, Minsk 220006, Belarus.

Published by the American Physical Society under the terms of the Creative Commons Attribution 4.0 International license. Further distribution of this work must maintain attribution to the author(s) and the published article's title, journal citation, and DOI. Funded by SCOAP ${ }^{3}$.
}

possibility of taking into account the effects of longitudinal polarization in variables of the experiment with necessary cutoffs.

Recently, interest in the elastic muon-electron scattering has increased due to high energy ( $\mu$ TRISTAN, KEK) and low energy (MUonE, CERN) experiments.

The new $\mu-e$ collider experiments $\mu$ TRISTAN with the energies $\left(E_{e^{-}}, E_{\mu^{+}}\right)=(30 \mathrm{GeV}, 1 \mathrm{TeV})$ and the beam polarizations with $P_{e^{-}}= \pm 0.7$ and $P_{\mu^{+}}= \pm 0.8$ are currently under consideration at KEK [5]. At such high energies, polarized $\mu-e$ scattering has not been thoroughly investigated.

Realistic conditions of the MUonE experiment [6,7] are the energy of incoming muon $E_{\mu}=150 \mathrm{GeV}$ in the laboratory system, which corresponds to the center-of-mass system (c.m.s.) energy $s=m_{e}^{2}+m_{\mu}^{2}+2 m_{e} E_{\mu} \approx(405 \mathrm{MeV})^{2}$ (see also proposals $[8,9]$ for new physics searches).

Further development of the most advanced theoretical support at the NLO and partly NNLO levels by MC codes for this experiment is provided by three scientific groups: ([10-13]), ([14,15]), and ([16,17]). A review of the current state of the theoretical support related to the proposed MUonE experiment and a discussion of further required steps can be found in [18].

The main result of this work is an independent calculation of the complete one-loop electroweak radiation corrections (RCs) to elastic muon-electron scattering,

$$
\begin{aligned}
& \mu^{+}\left(p_{1}, \chi_{1}\right)+e^{-}\left(p_{2}, \chi_{2}\right) \\
& \quad \rightarrow e^{-}\left(p_{3}, \chi_{3}\right)+\mu^{+}\left(p_{4}, \chi_{4}\right)\left(+\gamma\left(p_{5}, \chi_{5}\right)\right),
\end{aligned}
$$




$$
\begin{aligned}
& \mu^{-}\left(p_{1}, \chi_{1}\right)+e^{-}\left(p_{2}, \chi_{2}\right) \\
& \quad \rightarrow e^{-}\left(p_{3}, \chi_{3}\right)+\mu^{-}\left(p_{4}, \chi_{4}\right)\left(+\gamma\left(p_{5}, \chi_{5}\right)\right),
\end{aligned}
$$

with arbitrary polarizations of initial particles ( $\chi$ corresponds the helicity of the particles).

We perform a comparison for the hard bremsstrahlung cross section and check the numerical dependence of Born and corrected cross sections on the muon and electron polarization degrees. We present results for low energy in conditions of the MUonE experiment. To verify the effects of weak interactions, we present numerical results also for the c.m.s. energy $\sqrt{s}=250 \mathrm{GeV}$. Here we also computed leading higher-order (h.o.) corrections of the $\mathcal{O}\left(G_{\mu}^{2}\right)$ and $\mathcal{O}\left(G_{\mu} \alpha_{s}\right)$ orders through the parameters $\Delta \rho$ and $\Delta \alpha$. Our results can be considered as a preliminary glance at electroweak and polarization effects a new high-energy muon-electron collider [5].

This article is organized as follows. Section II contains preliminary remarks and the general notation. We describe the methodology for calculating polarized cross sections at the complete one-loop EW level within the helicity approach. Section III gives a description of leading h.o. EW corrections in $\mathcal{O}\left(G_{\mu}^{2}\right)$ and $\mathcal{O}\left(G_{\mu} \alpha_{s}\right)$ orders. In Sec. IV, we collect numerical results with various polarization degrees for total and differential cross sections as well as for relative corrections for high and low energies. The last Sec. V contains discussion and conclusions.

\section{EW ONE-LOOP RADIATIVE CORRECTIONS}

With the help of the computer system SANC [19], we have calculated the complete one-loop electroweak radiative corrections to a wide class of processes; see the review [20] and references therein. Recently, the system has been upgraded [21,22] in order to take into account possible longitudinal polarization of the initial particles. All calculations in the system can now be performed within the helicity amplitude formalism taking into account the initial and final state fermion masses. So the SANC system provides a solid framework to access polarization effects at the one-loop level and study various effects; for example, the system allows one to separate the effects due to QED, weak radiative corrections, and some higher-order contributions, to study radiative corrections in the $\alpha(0)$ and $G_{\mu}$ EW schemes.

A covariant amplitude (CA) corresponds to the result of the straightforward standard calculation by means of the SANC programs and procedures of all diagrams contributing to a given process at the tree (Born) and one-loop levels. In the SANC approach, we exploit the fact that the calculated one-loop form factors (FF) for the process $f \bar{f} f \bar{f} \rightarrow 0$ can be turned to any other channel. We describe the CA for this process decomposed on a massive basis with the so-called $\gamma, Q Q, Q L, L Q, L L, L D$, and $Q D$ contributions.
It corresponds to six Dirac structures; see [23]. They are labeled according to their structures. We have the same oneloop FFs for Bhabha scattering $e^{+} e^{-} \rightarrow e^{-} e^{+}, s$ channel $e^{+} e^{-} \rightarrow \mu^{-} \mu^{+}\left(\tau^{-} \tau^{+}\right)$, elastic $\mu-e$ scattering $\mu^{ \pm} e^{-} \rightarrow$ $e^{-} \mu^{ \pm}$, and Møller scattering $e^{-} e^{-} \rightarrow e^{-} e^{-}$. Form factors differ only by the corresponding permutations of their arguments $s, t, u$. The description of their implementation for Bhabha scattering and $s$ channel annihilation processes was presented in earlier articles [21,22].

The complete one-loop cross section of the process can be split into four parts,

$\sigma^{\text {one-loop }}=\sigma^{\text {Born }}+\sigma^{\text {virt }}(\lambda)+\sigma^{\text {soft }}(\lambda, \omega)+\sigma^{\text {hard }}(\omega)$,

where $\sigma^{\text {Born }}$ is the Born cross section, $\sigma^{\text {virt }}$ is the contribution of virtual (loop) corrections, and $\sigma^{\text {soft(hard) }}$ is the soft (hard) photon emission contribution (the hard photon energy $E_{\gamma}>\omega$ ). Auxiliary parameters $\lambda$ ("photon mass") and $\omega$ are canceled out after summation. Note that in calculations of one-loop RCs, we can separate QED and pure weak interaction effects.

In SANC, all one-loop contributions, i.e., the contributions of the virtual part as well as soft and hard bremsstrahlung radiation, are realized within the helicity amplitude (HA) approach as in several other modern codes for theoretical support of experiments; see, e.g., [24]. In HA, we keep the full dependence on the muon masses. As an additional bonus of the HA approach, we implement a procedure to compute the cross section with any polarization of the initial and final particles.

Typical SANC results for FF are given in terms of only scalar Passarino-Veltman (PV) functions [25]. In FF calculations at the one-loop level, we keep all masses (without any approximation), but in this study, we neglect contributions suppressed by the ratio $m_{e}^{2} / Q^{2}$ in the process cross section.

\section{A. Differential cross section}

To study the case of longitudinal polarization with degrees of $P_{e^{+}}$and $P_{e^{-}}$, we calculate helicity amplitudes and make a formal application of Eq. (1.15) from [26,27],

$$
\sigma\left(P_{e^{+}}, P_{e^{-}}\right)=\frac{1}{4} \sum_{\chi_{1}, \chi_{2}}\left(1+\chi_{1} P_{e^{+}}\right)\left(1+\chi_{2} P_{e^{-}}\right) \sigma_{\chi_{1} \chi_{2}},
$$

where $\chi_{1(2)}=-1(+1)$ corresponds to the particle $i$ with left (right) helicity.

The virtual (Born) cross section of the $\mu^{ \pm} e^{-} \rightarrow e^{-} \mu^{ \pm}$ process can be written as follows:

$$
\frac{d \sigma_{\chi_{1} \chi_{2}}^{\mathrm{virt}(\mathrm{Born})}}{d \cos \vartheta_{e}}=\pi \alpha^{2} \frac{1}{2 s}\left|\mathcal{H}_{\chi_{1} \chi_{2}}^{\mathrm{virt}(\text { Born })}\right|^{2},
$$


where

$$
\left|\mathcal{H}_{\chi_{1} \chi_{2}}^{\text {virt(Born) }}\right|^{2}=\sum_{\chi_{3}, \chi_{4}}\left|\mathcal{H}_{\chi_{1} \chi_{2} \chi_{3} \chi_{4}}^{\text {virt(Bon) }}\right|^{2}
$$

$\vartheta_{e}$ is the angle between the initial (anti)muon $\mu^{ \pm}$and final electron $e^{-}$.

The soft photon bremsstrahlung contributions are factorized at the Born cross section; they are given below.

The cross section for the hard photon bremsstrahlung reads

$$
\begin{aligned}
& \frac{d \sigma_{\chi_{1} \chi_{2}}^{\text {hard }}}{d s^{\prime} d \cos \theta_{4} d \phi_{4} d \cos \theta_{5}} \\
& =\alpha^{3} \frac{s-s^{\prime}}{128 \pi s s^{\prime}} \frac{\sqrt{\lambda\left(s^{\prime}, m_{\mu}^{2}, m_{e}^{2}\right)}}{\sqrt{\lambda\left(s, m_{\mu}^{2}, m_{e}^{2}\right)}}\left|\mathcal{H}_{\chi_{1} \chi_{2}}^{\text {hard }}\right|^{2},
\end{aligned}
$$

where $s^{\prime}=\left(p_{3}+p_{4}\right)^{2}, \lambda(x, y, z)$ is the Källén (triangle) function, and

$$
\left|\mathcal{H}_{\chi_{1} \chi_{2}}^{\text {hard }}\right|^{2}=\sum_{\chi_{3}, \chi_{4}, \chi_{5}}\left|\mathcal{H}_{\chi_{1} \chi_{2} \chi_{3} \chi_{4} \chi_{5}}^{\text {hard }}\right|^{2} .
$$

Here, $\theta_{5}$ is the angle between three momenta of the photon and electron, $\theta_{4}$ is the angle between three momenta of the (anti)muon $\mu^{ \pm}$and photon in the rest frame of the $\left(\mu^{ \pm} e^{-}\right)$ compound, $\phi_{4}$ is the azimuthal angle of the (anti)muon $\mu^{ \pm}$ in the same frame.

\section{B. Helicity amplitudes for the Born and virtual part}

For both channels, there are eight nonvanishing independent HAs which depend on kinematic variables, coupling constants, and six scalar FFs in the $L Q D$-basis for $Z$ boson and one for $\gamma$ exchange.

The HAs for $\mu^{+}$channel have the following form:

$$
\begin{aligned}
& \mathcal{H}_{\text {干干干干 }}=\frac{1}{t}\left\{s^{-}\left(2-c^{+} r_{s}\right) \tilde{F}_{\gamma}+\chi_{Z}(t)\left[s^{-}\left(-2 c^{+} r_{s} \tilde{F}_{L L}+\left(2-c^{+} r_{s}\right)\left(\tilde{F}_{Q Q}+\tilde{F}_{L Q}+\tilde{F}_{Q L}\right)-2 m_{\mu}^{2} c^{-}\left(\tilde{F}_{L D}+\tilde{F}_{Q D}\right)\right)\right.\right. \\
& \left.\left. \pm \sqrt{\lambda_{\mu e}}\left(-2 c^{+} r_{s} \tilde{F}_{L L}+\left(2-c^{+} r_{s}\right) \tilde{F}_{L Q}-\left(2+c^{+} r_{s}\right) \tilde{F}_{Q L}-2 c^{-} m_{\mu}^{2} \tilde{F}_{L D}\right)\right]\right\} \text {, } \\
& \mathcal{H}_{\mp-- \pm}=\sin \vartheta_{e} \frac{m_{\mu}}{\sqrt{s} t}\left\{s^{-} \tilde{F}_{\gamma}+\chi_{Z}(t)\left[s^{-}\left(2 \tilde{F}_{L L}+\tilde{F}_{Q Q}+\tilde{F}_{L Q}+\tilde{F}_{Q L}-s^{+}\left(\tilde{F}_{L D}+\tilde{F}_{Q D}\right)\right)\right.\right. \\
& \left.\left.+\sqrt{\lambda}_{\mu e}\left(2 \tilde{F}_{L L}+\tilde{F}_{L Q}+\tilde{F}_{Q L}-s^{+} \tilde{F}_{L D}\right)\right]\right\} \\
& \mathcal{H}_{\mp++ \pm}=-\sin \vartheta_{e} \frac{m_{\mu}}{\sqrt{s} t}\left\{s^{-} \tilde{F}_{\gamma}+\chi_{Z}(t)\left[s^{-}\left(2 \tilde{F}_{L L}+\tilde{F}_{Q Q}+\tilde{F}_{L Q}+\tilde{F}_{Q L}-s^{+}\left(\tilde{F}_{L D}+\tilde{F}_{Q D}\right)\right)\right.\right. \\
& \left.\left.-\sqrt{\lambda}_{\mu e}\left(2 \tilde{F}_{L L}+\tilde{F}_{L Q}+\tilde{F}_{Q L}-s^{+} \tilde{F}_{L D}\right)\right]\right\} \text {. } \\
& \mathcal{H}_{\mp \pm \pm \mp}=-\frac{c^{-}}{t}\left\{s^{-} \tilde{F}_{\gamma}+\chi_{Z}(t)\left[s^{-}\left(2 \tilde{F}_{L L}+\tilde{F}_{Q Q}+\tilde{F}_{L Q}+\tilde{F}_{Q L}-2 m_{\mu}^{2}\left(\tilde{F}_{L D}+\tilde{F}_{Q D}\right)\right)\right.\right. \\
& \left.\left.\mp \sqrt{\lambda}_{\mu e}\left(2 \tilde{F}_{L L}+\tilde{F}_{L Q}+\tilde{F}_{Q L}-2 m_{\mu}^{2} \tilde{F}_{L D}\right)\right]\right\} \text {, }
\end{aligned}
$$

where $\lambda_{\mu e}=\lambda\left(s, m_{\mu}^{2}, m_{e}^{2}\right)$.

The HAs for $\mu^{-}$- channel have the following form:

$$
\begin{aligned}
& \mathcal{H}_{ \pm \pm \pm \pm}=-\frac{1}{t}\left\{s^{-}\left(2-c^{+} r_{s}\right) \tilde{F}_{\gamma}+\chi_{Z}(t)\left[s^{-}\left(4 \tilde{F}_{L L}+\left(2-c^{+} r_{s}\right)\left(\tilde{F}_{Q Q}+\tilde{F}_{L Q}+\tilde{F}_{Q L}\right)-2 m_{\mu}^{2} c^{-}\left(\tilde{F}_{L D}+\tilde{F}_{Q D}\right)\right)\right.\right. \\
& \left.\left.\mp \sqrt{\lambda}_{\mu e}\left(4\left(\tilde{F}_{Q L}+\tilde{F}_{L L}\right)+\left(2-c^{+} r_{s}\right)\left(\tilde{F}_{L Q}-\tilde{F}_{Q L}\right)-2 m_{\mu}^{2} c^{-} \tilde{F}_{L D}\right)\right]\right\} \text {, } \\
& \mathcal{H}_{- \pm \pm+}=-\sin \vartheta_{e} \frac{m_{\mu}}{t \sqrt{s}}\left\{s^{-} \tilde{F}_{\gamma}+\chi_{Z}(t)\left[s^{-}\left(\tilde{F}_{Q Q}+\tilde{F}_{L Q}+\tilde{F}_{Q L}-s^{+}\left(\tilde{F}_{L D}+\tilde{F}_{Q D}\right)\right) \mp \sqrt{\lambda}_{\mu e}\left(\tilde{F}_{L Q}-\tilde{F}_{Q L}-s^{+} \tilde{F}_{L D}\right)\right]\right\} \text {, } \\
& \mathcal{H}_{+ \pm \pm-}=\sin \vartheta_{e} \frac{m_{\mu}}{t \sqrt{s}}\left\{s^{-} \tilde{F}_{\gamma}+\chi_{Z}(t)\left[s^{-}\left(\tilde{F}_{Q Q}+\tilde{F}_{L Q}+\tilde{F}_{Q L}-s^{+}\left(\tilde{F}_{L D}+\tilde{F}_{Q D}\right)\right) \mp \sqrt{\lambda}_{\mu e}\left(\tilde{F}_{L Q}-\tilde{F}_{Q L}-s^{+} \tilde{F}_{L D}\right)\right]\right\} \text {, } \\
& \mathcal{H}_{ \pm \mp \mp \pm}=-\frac{c^{-}}{t}\left\{s^{-} \tilde{F}_{\gamma}+\chi_{Z}(t)\left[s^{-}\left(\tilde{F}_{Q Q}+\tilde{F}_{L Q}+\tilde{F}_{Q L}-2 m_{\mu}^{2}\left(\tilde{F}_{L D}+\tilde{F}_{Q D}\right)\right) \pm \sqrt{\lambda}_{\mu e}\left(\tilde{F}_{L Q}-\tilde{F}_{Q L}-2 m_{\mu}^{2} \tilde{F}_{L D}\right)\right]\right\}
\end{aligned}
$$


with $s^{-}=s-m_{\mu}^{2}, s^{+}=s+m_{\mu}^{2}$, and $\chi_{Z}(t)$ being the $Z / \gamma$ propagator ratio,

$$
\chi_{Z}(t)=\frac{1}{4 s_{W}^{2} c_{W}^{2}} \frac{t}{t-M_{Z}^{2}}
$$

For both channels, the other six HAs are expressed through the above ones as follows: $\mathcal{H}_{---+}=\mathcal{H}_{+---}$, $\mathcal{H}_{--++}=\mathcal{H}_{++--}, \mathcal{H}_{-+--}=\mathcal{H}_{--+-}, \mathcal{H}_{-+-+}=\mathcal{H}_{+-+-}$, $\mathcal{H}_{-+++}=\mathcal{H}_{+++-}, \mathcal{H}_{+-++}=\mathcal{H}_{++-+}$.

The helicity indices denote the signs of the fermion spin projections to their momenta $p_{1}, p_{2}, p_{3}, p_{4}$, respectively. The notation,

$$
c^{ \pm}=1 \pm \cos \vartheta_{e}
$$

was introduced. The electron scattering angle $\vartheta_{e}$ is related to the Mandelstam variables $t=\left(p_{-}-p_{4}\right)^{2}$ and $u=\left(p_{2}-p_{3}\right)^{2}$,

$$
\cos \vartheta_{\mu}=\left[s(u-t)-\left(m_{\mu}^{2}-m_{e}^{2}\right)^{2}\right] / \lambda_{\mu e}
$$

Note that the tilded form factors absorb couplings, which leads to compactification of the formulas for the HAs. The expressions for the tilded form factors are

$$
\begin{gathered}
\tilde{F}_{\gamma}=Q_{e} Q_{\mu} F_{\gamma}, \\
\tilde{F}_{L L}=I_{e}^{(3)} I_{\mu}^{(3)} F_{L L}, \\
\tilde{F}_{L Q}=I_{e}^{(3)} \delta_{\mu} F_{L Q}, \\
\tilde{F}_{Q L}=\delta_{e} I_{\mu}^{(3)} F_{Q L}, \\
\tilde{F}_{Q Q}=\delta_{e} \delta_{\mu} F_{Q Q}, \\
\tilde{F}_{L D}=I_{e}^{(3)} I_{\mu}^{(3)} F_{L D}, \\
\tilde{F}_{Q D}=\delta_{e} I_{\mu}^{(3)} F_{Q D},
\end{gathered}
$$

with the coupling constants,

$$
\begin{aligned}
I_{f}^{(3)}, \quad \sigma_{f} & =v_{f}+a_{f}, \quad \delta_{f}=v_{f}-a_{f}, \\
s_{W} & =\frac{e}{g}, \quad c_{W}=\frac{M_{\mathrm{W}}}{M_{\mathrm{Z}}} .
\end{aligned}
$$

In order to get HAs for the Born level, one should set $F_{\gamma, L L, L Q, Q L, Q Q}=1$ and $F_{L D, Q D}=0$.

\section{The soft photon emission bremsstrahlung}

The soft photon contribution contains the infrared divergences and compensates the corresponding divergences of the one-loop virtual QED corrections. This soft photon bremsstrahlung correction can be calculated analytically, and it is factorized at the Born cross section. In this case, the polarization dependence is contained only in the Born cross section $\sigma^{\text {Born, }}$

$$
\begin{aligned}
& \sigma^{\mathrm{soft}, \mu-\mathrm{leg}}=-Q_{\mu}^{2} \frac{\alpha}{\pi} \sigma^{\text {Born }}\left\{\ln \left(\frac{4 \omega^{2}}{\lambda^{2}}\right)+\frac{s+m_{\mu}^{2}}{s-m_{\mu}^{2}} \ln \left(r_{s}\right)-\frac{1+2 r_{t}}{\sqrt{1+4 r_{t}}}\left[\ln \left(a_{14}\right) \ln \left(\frac{4 \omega^{2}}{\lambda^{2}}\right)\right.\right. \\
& \left.\left.+\mathrm{Li}_{2}\left(1-\frac{a_{14} x_{\mu}}{v_{14}}\right)-\mathrm{Li}_{2}\left(1-\frac{x_{\mu}}{v_{14}}\right)+\mathrm{Li}_{2}\left(1-\frac{a_{14}}{v_{14} x_{\mu}}\right)-\mathrm{Li}_{2}\left(1-\frac{1}{v_{14} x_{\mu}}\right)\right]\right\} \text {, } \\
& \sigma^{\text {soft,ifi }}=-Q_{e} Q_{\mu} \frac{\alpha}{\pi} \sigma^{\text {Born }}\left\{2 \ln \left(x_{\mu} x_{e}\right) \ln \left(\frac{4 \omega^{2}}{\lambda^{2}}\right)+\operatorname{Li}_{2}\left(1-x_{\mu}^{2}\right)+\operatorname{Li}_{2}\left(1-x_{e}^{2}\right)-\operatorname{Li}_{2}\left(1-\frac{1}{x_{\mu}^{2}}\right)-\operatorname{Li}_{2}\left(1-\frac{1}{x_{e}^{2}}\right)\right. \\
& -\ln \left(a_{13}\right) \ln \left(\frac{4 \omega^{2}}{\lambda^{2}}\right)-\mathrm{Li}_{2}\left(1-\frac{a_{13} x_{\mu}}{v_{13}}\right)-\mathrm{Li}_{2}\left(1-\frac{x_{e}}{v_{13}}\right)-\mathrm{Li}_{2}\left(1-\frac{a_{13}}{v_{13} x_{\mu}}\right)+\mathrm{Li}_{2}\left(1-\frac{1}{v_{13} x_{e}}\right) \\
& \left.-\ln \left(a_{24}\right) \ln \left(\frac{4 \omega^{2}}{\lambda^{2}}\right)-\mathrm{Li}_{2}\left(1-\frac{a_{24} x_{e}}{v_{24}}\right)+\mathrm{Li}_{2}\left(1-\frac{x_{\mu}}{v_{24}}\right)-\mathrm{Li}_{2}\left(1-\frac{a_{24}}{v_{24} x_{e}}\right)+\mathrm{Li}_{2}\left(1-\frac{1}{v_{24} x_{\mu}}\right)\right\} \text {, } \\
& \sigma^{\text {soft,e-leg }}=-Q_{e}^{2} \frac{\alpha}{\pi} \sigma^{\text {Born }}\left\{\ln \left(\frac{4 \omega^{2}}{\lambda^{2}}\right)+\ln \left(\frac{m_{e}^{2}}{s\left(1-r_{s}^{2}\right)}\right)-\ln \left(a_{23}\right) \ln \left(\frac{4 \omega^{2}}{\lambda^{2}}\right)\right. \\
& \left.-\mathrm{Li}_{2}\left(1-\frac{a_{23} x_{e}}{v_{23}}\right)+\mathrm{Li}_{2}\left(1-\frac{x_{e}}{v_{23}}\right)-\mathrm{Li}_{2}\left(1-\frac{a_{23}}{v_{23} x_{e}}\right)+\mathrm{Li}_{2}\left(1-\frac{1}{v_{23} x_{e}}\right)\right\} \text {. }
\end{aligned}
$$


With the definitions,

$$
\begin{gathered}
x_{\mu}=\frac{\sqrt{s}}{m_{\mu}}, \quad x_{e}=\frac{\sqrt{s}}{m_{e}}\left(1-r_{s}\right), \\
a_{14}=\frac{1}{2 m_{\mu}^{2}}\left(2 m_{\mu}^{2}+t+\sqrt{t^{2}+4 m_{\mu}^{2} t}\right), \\
v_{14}=\frac{m_{\mu}}{\sqrt{s}\left(1+r_{s}\right)}\left(a_{14}+1\right), \\
a_{23}=\frac{t}{m_{e}^{2}}, \quad v_{23}=\frac{m_{e}}{\sqrt{s}\left(1-r_{s}\right)}\left(a_{23}+1\right), \\
a_{13}=\frac{\left(u+m_{\mu}^{2}\right)}{m_{e} m_{\mu}}, \quad a_{13}^{2} \\
v_{13}=\frac{r_{s}}{\sqrt{s}\left(a_{13}\left(1+r_{s}\right) / m_{\mu}-\left(1-r_{s}\right) / m_{e}\right)}, \\
a_{24}=a_{13}, \\
v_{24}=\frac{a_{24}^{2}}{\sqrt{s}\left(a_{24}\left(1-r_{s}\right) / m_{e}-\left(1+r_{s}\right) / m_{\mu}\right)},
\end{gathered}
$$

where $r_{I}=m_{\mu}^{2} / I$ and $I=s, t$.

\section{Helicity amplitudes for hard photon bremsstrahlung}

We use the SANC spinor amplitude module for the $e^{+} e^{-} l^{+} l^{-} \gamma \rightarrow 0\left(\sum p_{i}=0\right)$ process in any of the $s, t$, or $u$ channels, where 0 stands for vacuum and all masses are not neglected.

The full expression for the photon bremsstrahlung amplitude of the process under investigation can be divided into two terms,

$$
\mathrm{A}_{\ldots \chi_{i} \ldots}=2 \sqrt{2}\left(Q_{\mu} A_{\ldots \chi_{i} \ldots}^{\mu}+Q_{e} A_{\ldots \chi_{i} \ldots}^{e}\right) .
$$

Each term corresponds to a gauge-invariant diagram subset: $A^{\mu}$ is the amplitude for radiation off the muon line (MSR) and $A^{e}$ for radiation off the electron line (ESR).

There exists the following crossing symmetry relation between MSR and ESR amplitudes:

$$
\begin{aligned}
& A^{\mu}{ }_{\chi_{1} \chi_{4} \chi_{3} \chi_{2} \chi_{5}}\left(p_{1}, p_{4}, p_{3}, p_{2}, p_{5}\right) \\
& \quad=A^{e}{ }_{\chi_{3} \chi_{2} \chi_{1} \chi_{4} \chi_{5}}\left(p_{3}, p_{2}, p_{1}, p_{4}, p_{5}\right) \text { and } m_{\mu} \leftrightarrow m_{e} .
\end{aligned}
$$

The explicitly gauge invariant form of the amplitude is obtained in [22] and implemented as a SANC module,

$$
\begin{aligned}
\sqrt{2} A_{\chi_{1} \chi_{2} \chi_{3} \chi_{4} \chi_{5}}^{e}= & \frac{\operatorname{Tr}\left[\not \not_{1} \not \not_{2} \mathbf{F}_{5}\right]}{z_{1} z_{2}} \bar{v}_{1} \phi_{34} u_{2} \\
& -\frac{\bar{v}_{1} \mathbf{F}_{5} \phi_{34} u_{2}}{z_{1}}-\frac{\bar{v}_{1} \phi_{34} \mathbf{F}_{5} u_{2}}{z_{2}},
\end{aligned}
$$

with abbreviations $z_{i}=2 p_{i} \cdot p_{5}, u_{i} \equiv u^{\chi_{i}}\left(p_{i}\right)$ etc.

The polarization vector of a real photon appears only in the combination $\mathbf{F}_{5}=p_{5}^{\mu} \varepsilon_{5}^{\nu} \sigma_{\mu \nu}$. This is the familiar Maxwell bivector which is gauge invariant. We introduce also abbreviations for the following combinations of propagators and couplings constants:

$$
\begin{aligned}
\phi_{34} & =\frac{1}{2} \sum_{a, b= \pm 1} \mathcal{D}^{a b}\left(\bar{v}_{3} \gamma^{\mu} \gamma_{b} u_{4}\right) \gamma_{\mu} \gamma_{a}, \\
\mathcal{D}^{a b} & =\frac{Q_{e} Q_{l}}{s^{\prime}}+\frac{g_{e}^{a} g_{l}^{b}}{s^{\prime}-M_{Z}^{2}+i M_{Z} \Gamma_{Z}},
\end{aligned}
$$

where $g_{l}^{ \pm}$are the chiral couplings of the leptons $l$ to the vector boson $Z$.

We work in the chiral representation of gamma matrices and exploit Weyl spinors. Our notation is consistent with [28-30]. Below we use the following notation for the decomposition of Dirac spinors into Weyl ones:

$$
\begin{aligned}
& \not p=\left(\begin{array}{cc} 
& p_{A \dot{B}} \\
p^{\dot{A} B} &
\end{array}\right)=\left(\begin{array}{cc} 
& \check{p} \\
\hat{p} &
\end{array}\right), \\
& u=\left(\begin{array}{c}
u_{A} \\
u^{\dot{A}}
\end{array}\right)=\left(\begin{array}{l}
|u\rangle \\
\mid u]
\end{array}\right), \\
& \bar{u}=\left(\begin{array}{ll}
\bar{u}^{A}, & \bar{u}_{\dot{A}}
\end{array}\right)=\left(\begin{array}{ll}
\langle\bar{u}|, & {[\bar{u} \mid}
\end{array}\right), \\
& \mathbf{F}=\left(\begin{array}{cc}
\mathbf{F}_{A}{ }^{B} & \\
& \mathbf{F}_{\dot{B}}^{\dot{A}}
\end{array}\right)=\left(\begin{array}{ll}
\check{\mathbf{F}} & \\
& \hat{\mathbf{F}}
\end{array}\right) .
\end{aligned}
$$

Application of the Fiertz identities to the Pauli matrices yields

$$
\begin{aligned}
& \left.\hat{e}_{34}=\mid \bar{v}_{3}\right] \mathcal{D}^{++}\left\langle u_{4}|+| u_{4}\right] \mathcal{D}^{+-}\left\langle\bar{v}_{3}\right|, \\
& \check{e}_{34}=\left|u_{4}\right\rangle \mathcal{D}^{-+}\left[\bar{v}_{3}|+| \bar{v}_{3}\right\rangle \mathcal{D}^{--}\left[u_{4} \mid .\right.
\end{aligned}
$$

The HAs are not Lorentz-invariant objects (they are changed by boosts transverse to a momentum ray) and thus, rudimentarily depend on an experimental setup. However, one expects that the entire physical content of a reaction should depend only on a relative configuration of particles by analogy with the rigid body dynamics in a rotating reference frame. This type of description usually appears to be most economic one.

In order to factor out all information related to an experiment configuration, we must build a spin basis in terms of the given problem's momenta. Our investigations show that one of the most economic choices is to put the polarization vector of fermion $n_{i}$ with $i=1, \ldots, 4$ into the 
same two-plane with its momentum $p_{i}$ and momentum of photon $p_{5}$. Each two-plane contains two light-like vectors: photon momentum and the other one denoted by $k_{i}$. The explicit expressions for $k_{i}$ can be found in [31]. Then in the photon basis, we have

$$
\begin{aligned}
& \left|u_{i}^{+}\right\rangle=\left|v_{i}^{-}\right\rangle=\left|\bar{u}_{i}^{-}\right\rangle=\left|\bar{v}_{i}^{+}\right\rangle=\left|k_{i}\right\rangle \equiv|i\rangle, \\
& \left.\left.\left.\left.\left.\left.\mid u_{i}^{-}\right]=\mid v_{i}^{+}\right]=\mid \bar{u}_{i}^{+}\right]=\mid \bar{v}_{i}^{-}\right]=\mid k_{i}\right] \equiv \mid i\right], \\
& \left.\left.\left.\left.\left.\mid u_{i}^{+}\right]=-\mid v_{i}^{-}\right]=-\mid \bar{u}_{i}^{-}\right]=\mid \bar{v}_{i}^{+}\right]=-\mid 5\right] \varsigma_{i}^{*}, \\
& \left|u_{i}^{-}\right\rangle=-\left|v_{i}^{+}\right\rangle=-\left|\bar{u}_{i}^{+}\right\rangle=\left|\bar{v}_{i}^{-}\right\rangle=|5\rangle \varsigma_{i},
\end{aligned}
$$

with $\varsigma_{i}=m_{i} /\langle i \mid 5\rangle$, where we identify $k_{5} \equiv p_{5}$.

A Maxwell bivector has a factorized form in the spinor notation: $\check{\mathbf{F}}_{5}^{+}=\sqrt{2}|5\rangle\left\langle 5\left|, \quad \hat{\mathbf{F}}_{5}^{-}=\sqrt{2}\right| 5\right]\left[5 \mid, \check{\mathbf{F}}_{5}^{-}=\hat{\mathbf{F}}_{5}^{+}=0\right.$, which allows us to organize the amplitude in terms of blocks,

$A_{\chi_{1} \chi_{2} \chi_{3} \chi_{4} \chi_{5}}^{e}=\mathcal{S}_{\chi_{5}} \mathcal{B}_{\chi_{1} \chi_{2} \chi_{3} \chi_{4}}-\mathcal{C}_{\chi_{1} \chi_{5}}^{1} \mathcal{G}_{\chi_{2} \chi_{3} \chi_{4} \chi_{5}}^{2}-\mathcal{C}_{\chi_{2} \chi_{5}}^{2} \mathcal{G}_{\chi_{1} \chi_{3} \chi_{4} \chi_{5}}^{1}$,

where

$$
\begin{aligned}
\mathcal{B}_{\chi_{1} \chi_{2} \chi_{3} \chi_{4}} & =\left[\bar{v}_{1}\left|\hat{e}_{34}\right| u_{2}\right\rangle+\left\langle\bar{v}_{1}\left|\check{e}_{34}\right| u_{2}\right], \\
\mathcal{G}_{\chi_{1} \chi_{3} \chi_{4} \pm}^{1} & =\left[\begin{array}{l}
{\left[\bar{v}_{1}\left|\hat{e}_{34}\right| 5\right\rangle} \\
\left\langle\bar{v}_{1}\left|\check{e}_{34}\right| 5\right]
\end{array}\right], \\
\mathcal{G}_{\chi_{2} \chi_{3} \chi_{4} \pm}^{2} & =\left[\begin{array}{c}
\left\langle 5\left|\check{e}_{34}\right| u_{2}\right] \\
{\left[5\left|\hat{e}_{34}\right| u_{2}\right\rangle}
\end{array}\right], \\
\mathcal{S}_{\chi_{5}} & =-\left[\frac{[1 \mid 2]}{[1 \mid 5][2 \mid 5]}, \frac{\langle 1 \mid 2\rangle}{\langle 1 \mid 5\rangle\langle 2 \mid 5\rangle}\right], \\
\mathcal{C}_{\chi_{1} \chi_{5}}^{1} & =\left[\begin{array}{rr}
{[5 \mid 1]} & \langle 5 \mid 1\rangle
\end{array}\right]^{-1}, \\
\mathcal{C}_{\chi_{2} \chi_{5}}^{2} & =\left[\begin{array}{rr}
{[2 \mid 5]} & \langle 2 \mid 5\rangle
\end{array}\right]^{-1} .
\end{aligned}
$$

We are going to evaluate the amplitude only for positive photon helicity because the case of negative one can be easily obtained with the help of the $C P$ symmetry,

$$
A_{\chi_{1} \chi_{2} \chi_{3} \chi_{4}-}=-\chi_{1} \chi_{2} \chi_{3} \chi_{4} A^{*}{ }_{-\chi_{1}-\chi_{2}-\chi_{3}-\chi_{4}+}
$$

with “+” $\leftrightarrow$ "-" in $\mathcal{D}^{a b}$. Below, we give all amplitudes with positive photon helicity,

$$
\begin{aligned}
& A_{---++}^{e}=-\mathcal{S}_{+}\langle 4 \mid 5\rangle\left(\mathcal{D}^{++}[3 \mid 1] \varsigma_{2}+\mathcal{D}^{-+}[3 \mid 2] \varsigma_{1}\right), \\
& A_{--+-+}^{e}=-\mathcal{S}_{+}\langle 3 \mid 5\rangle\left(\mathcal{D}^{+-}[4 \mid 1] \varsigma_{2}+\mathcal{D}^{--}[4 \mid 2] \varsigma_{1}\right), \\
& A^{e}{ }_{-+--+}=\mathcal{S}_{+}\langle 2 \mid 5\rangle\left(\mathcal{D}^{++}[3 \mid 1] \varsigma_{4}+\mathcal{D}^{+-}[4 \mid 1] \varsigma_{3}\right), \\
& A^{e}{ }_{+---+}=\mathcal{S}_{+}\langle 1 \mid 5\rangle\left(\mathcal{D}^{-+}[3 \mid 2] \varsigma_{4}+\mathcal{D}^{--}[4 \mid 2] \varsigma_{3}\right), \\
& A^{e}{ }_{--+++}=\mathcal{S}_{+}[5 \mid 1]\left(\mathcal{D}^{+-}\langle 3 \mid 5\rangle \varsigma_{2} \varsigma_{4}^{*}+\mathcal{D}^{++}\langle 4 \mid 5\rangle \varsigma_{2} \varsigma_{3}^{*}\right) \\
& +\mathcal{S}_{+}[5 \mid 2]\left(\mathcal{D}^{--}\langle 3 \mid 5\rangle \varsigma_{1} \varsigma_{4}^{*}+\mathcal{D}^{-+}\langle 4 \mid 5\rangle \varsigma_{1} \varsigma_{3}^{*}\right), \\
& \left.A^{e}{ }_{++--+}=\mathcal{S}_{+}\langle 1 \mid 5\rangle \mathcal{D}^{-+}[5 \mid 3] \varsigma_{2}^{*} \varsigma_{4}+\mathcal{D}^{--}[5 \mid 4] \varsigma_{2}^{*} \varsigma_{3}\right) \\
& +\mathcal{S}_{+}\langle 2 \mid 5\rangle\left(\mathcal{D}^{++}[5 \mid 3] \varsigma_{1}^{*} \varsigma_{4}+\mathcal{D}^{+-}[5 \mid 4] \varsigma_{1}^{*} \varsigma_{3}\right), \\
& A^{e}{ }_{-+-++}=\mathcal{S}_{+}\left(\mathcal{D}^{++}[3 \mid 1]\langle 2 \mid 4\rangle-\mathcal{D}^{-+}\langle 4 \mid 5\rangle[5 \mid 3] \varsigma_{1} \varsigma_{2}^{*}\right. \\
& \left.-\mathcal{D}^{+-}\langle 2 \mid 5\rangle[5 \mid 1] \varsigma_{3} \varsigma_{4}^{*}\right)+\mathcal{C}_{++}^{2} \mathcal{D}^{++}[3 \mid 1]\langle 4 \mid 5\rangle, \\
& A^{e}{ }_{-++-+}=\mathcal{S}_{+}\left(\mathcal{D}^{+-}[4 \mid 1]\langle 2 \mid 3\rangle-\mathcal{D}^{--}\langle 3 \mid 5\rangle[5 \mid 4] \varsigma_{1} \varsigma_{2}^{*}\right. \\
& \left.-\mathcal{D}^{++}\langle 2 \mid 5\rangle[5 \mid 1] \varsigma_{3}^{*} \varsigma_{4}\right)+\mathcal{C}_{++}^{2} \mathcal{D}^{+-}[4 \mid 1]\langle 4 \mid 5\rangle, \\
& A^{e}{ }_{+--++}=\mathcal{S}_{+}\left(\mathcal{D}^{-+}[3 \mid 2]\langle 1 \mid 4\rangle-\mathcal{D}^{++}\langle 4 \mid 5\rangle \mid[5 \mid 3] \varsigma_{1}^{*} \varsigma_{2}\right. \\
& \left.-\mathcal{D}^{--}\langle 1 \mid 5\rangle[5 \mid 2] \varsigma_{3} \varsigma_{4}^{*}\right)+\mathcal{C}_{++}^{1} \mathcal{D}^{-+}[3 \mid 2]\langle 4 \mid 5\rangle, \\
& A^{e}{ }_{+-+-+}=\mathcal{S}_{+}\left(\mathcal{D}^{--}[4 \mid 2]\langle 1 \mid 3\rangle-\mathcal{D}^{+-}\langle 3 \mid 5\rangle[5 \mid 4] \varsigma_{1}^{*} \varsigma_{2}\right. \\
& \left.-\mathcal{D}^{-+}\langle 1 \mid 5\rangle[5 \mid 2] \varsigma_{3}^{*} \varsigma_{4}\right)+\mathcal{C}_{++}^{1} \mathcal{D}^{--}[4 \mid 2]\langle 3 \mid 5\rangle, \\
& A^{e}{ }_{-++++}=-\mathcal{S}_{+}[5 \mid 1]\left(\mathcal{D}^{+-}\langle 2 \mid 3\rangle \varsigma_{4}^{*}+\mathcal{D}^{++}\langle 2 \mid 4\rangle \varsigma_{3}^{*}\right) \\
& -\mathcal{C}_{++}^{2}[5 \mid 1]\left(\mathcal{D}^{+-}\langle 3 \mid 5\rangle \varsigma_{4}^{*}+\mathcal{D}^{++}\langle 4 \mid 5\rangle \varsigma_{3}^{*}\right), \\
& A^{e}{ }_{+-+++}=-\mathcal{S}_{+}[5 \mid 2]\left(\mathcal{D}^{--}\langle 1 \mid 3\rangle \varsigma_{4}^{*}+\mathcal{D}^{-+}\langle 1 \mid 4\rangle \varsigma_{3}^{*}\right) \\
& -\mathcal{C}_{++}^{2}[5 \mid 2]\left(\mathcal{D}^{--}\langle 3 \mid 5\rangle \varsigma_{4}^{*}+\mathcal{D}^{-+}\langle 4 \mid 5\rangle \varsigma_{3}^{*}\right), \\
& A^{e}{ }_{++-++}=\mathcal{S}_{+}[5 \mid 3]\left(\mathcal{D}^{-+}\langle 1 \mid 4\rangle \varsigma_{2}^{*}+\mathcal{D}^{++}\langle 2 \mid 4\rangle \varsigma_{1}^{*}\right) \\
& +\langle 4 \mid 5\rangle[5 \mid 3]\left(\mathcal{C}_{++}^{1} \mathcal{D}^{-+} \varsigma_{2}^{*}+\mathcal{C}_{++}^{2} \mathcal{D}^{++} \varsigma_{1}^{*}\right), \\
& A^{e}{ }_{+++-+}=\mathcal{S}_{+}[5 \mid 4]\left(\mathcal{D}^{--}\langle 1 \mid 3\rangle \varsigma_{2}^{*}+\mathcal{D}^{+-}\langle 2 \mid 3\rangle \varsigma_{1}^{*}\right) \\
& +\langle 3 \mid 5\rangle[5 \mid 4]\left(\mathcal{C}_{++}^{1} \mathcal{D}^{--} \varsigma_{2}^{*}+\mathcal{C}_{++}^{2} \mathcal{D}^{+-} \varsigma_{1}^{*}\right), \\
& A^{e}{ }_{+++++}=A_{----+}^{e}=0 \text {. }
\end{aligned}
$$

To obtain HA $\mathcal{H}$ with a definite set of helicities, the basis-transformation matrices $C_{\xi_{i}}^{\chi_{i}}$ should be applied independently for each index $\chi$ of external particles whose polarization is not averaged,

$$
\mathcal{H}_{\ldots \xi_{i} \ldots}=C_{\xi_{1}}^{\chi_{1}} \ldots C_{\xi_{4}}^{\chi_{4}} \mathrm{~A}_{\ldots \chi_{i} \ldots}
$$

Explicit expressions for the matrices $C$ can be found in [32] and for our special case in [31]. Geometrically, they realize a Wigner rotation of the spin axis [33,34].

\section{LEADING HIGHER-ORDER ELECTROWEAK EFFECTS}

\section{A. The $\rho$ parameter}

The electroweak parameter $\rho$, introduced by Veltman [35], measures the relative strength of charged and neutral currents. The $\rho$ parameter is defined as the ratio of a neutral 
current amplitude to a charged current one at the zero momentum transfer; see, for example, [36],

$$
\rho=\frac{G_{N C}(0)}{G_{C C}(0)}=\frac{1}{1-\Delta \rho},
$$

where $G_{C C}(0)=G_{\mu}$ is the Fermi constant defined from the $\mu$ decay width, and the quantity $\Delta \rho$ is treated perturbatively,

$$
\Delta \rho=\Delta \rho^{(1)}+\Delta \rho^{(2)}+\ldots
$$

Expanding (27) up to quadratic terms $\Delta \rho^{2}$, we have

$$
\rho=1+\Delta \rho+\Delta \rho^{2}
$$

The leading in $G_{\mu} m_{t}^{2}$ NLO EW contribution to $\Delta \rho$ is explicitly given by

$$
\left.\Delta \rho^{(1)}\right|^{G_{\mu}}=3 X_{t}=\frac{3 \sqrt{2} G_{\mu} m_{t}^{2}}{16 \pi^{2}} .
$$

A large group of dominant radiative corrections can be absorbed into the shift of the $\rho$ parameter from its lowest order value $\rho_{\text {Born }}=1$. The major contributions of these groups are

$$
\Delta \rho=\Delta \rho_{X_{t}}+\Delta \rho_{\alpha \alpha_{s}}+\Delta \rho_{X_{t} \alpha_{s}^{2}}+\ldots
$$

We follow the prescription introduced in Refs. [36,37] and later well described in Refs. [38,39].

At the two-loop level, the quantity $\Delta \rho$ contains two contributions,

$$
\begin{aligned}
\Delta \rho= & N_{c} X_{t}\left[1+\rho^{(2)}\left(M_{\mathrm{H}}^{2} / m_{t}^{2}\right) X_{t}\right] \\
& \times\left[1-\frac{2 \alpha_{s}\left(M_{\mathrm{Z}}^{2}\right)}{9 \pi}\left(\pi^{2}+3\right)\right] .
\end{aligned}
$$

They consist of the following:

(i) The two-loop EW part at $\mathcal{O}\left(G_{\mu}^{2}\right)$, the second term in the first square brackets $[36,37,40]$ with $\rho^{(2)}$ given in Eq. (12) of [37] [actually, after the Higgs boson discovery and determination of its mass, it has become sufficient to use the low Higgs mass asymptotics, Eq. (15), of [37]];

(ii) The mixed two-loop EW $\otimes \mathrm{QCD}$ at $\mathcal{O}\left(G_{\mu} \alpha_{s}\right)$, the second term in the second square brackets; see Refs. [41,42] for further details.

\section{B. Implementation of the $\Delta \alpha$ and $\Delta \rho$ parameters}

The leading higher-order effects are usually parametrized by $\Delta \alpha$ and $\Delta \rho$ and their two-loop contributions can be included in a straightforward way.

The corrections induced by the running of $\alpha$ can be included by resummation the NLO EW $\Delta \alpha$ parameter,

$$
\alpha\left(Q^{2}\right)=\frac{\alpha(0)}{1-\Delta \alpha\left(Q^{2}\right)} .
$$

Here, $Q^{2}$ is the scale which characterizes the evolution of the EW coupling. In the case of elastic $\mu-e$ scattering, $Q^{2}=t$ where $t$ is the Mandelstam variable.

To implement the $\Delta \rho$ parameter, we introduce intermediate vector boson propagators $\sim 1 /\left(Q^{2}+M_{V}^{2}\right)$, into Eq. (27) and derive the following definition:

$$
\rho=\frac{M_{\mathrm{W}}^{2}}{\bar{c}_{W}^{2} M_{\mathrm{Z}}^{2}},
$$

where we have introduced a new parameter $\bar{c}_{W}^{2}$ to distinguish from the usual $c_{W}^{2}$ for which we maintain the meaning $c_{W}^{2}=M_{\mathrm{W}}^{2} / M_{\mathrm{Z}}^{2}$ to be valid to all perturbative orders. At the lowest order (LO),

$$
\rho^{(0)}=\frac{M_{\mathrm{W}}^{2}}{c_{W}^{2} M_{\mathrm{Z}}^{2}}=1 .
$$

From Eq. (34), we have

$$
\bar{c}_{W}^{2}=\frac{M_{\mathrm{W}}^{2}}{\rho M_{\mathrm{Z}}^{2}}=(1-\Delta \rho) c_{W}^{2}, \quad \bar{s}_{W}^{2}=s_{W}^{2}+\Delta \rho c_{W}^{2} .
$$

Equations (33) and (36) allow us to introduce the leading higher-order effects via the following replacements:

$$
\begin{gathered}
\alpha(0) \rightarrow \alpha(t)=\alpha(0)\left[1+\Delta \alpha(t)+\Delta \alpha(t)^{2}\right], \\
s_{W}^{2} \rightarrow \bar{s}_{W}^{2} \equiv s_{W}^{2}\left(1+\frac{c_{W}^{2}}{s_{W}^{2}} \Delta \rho\right) \\
c_{W}^{2} \rightarrow \bar{c}_{W}^{2} \equiv 1-\bar{s}_{W}^{2}=(1-\Delta \rho) c_{W}^{2},
\end{gathered}
$$

into the Born cross section with $\alpha(0)$ EW coupling. The obtained recipe allows one to reproduce correctly the terms up to $\mathcal{O}\left(\Delta \rho^{2}\right)$ and $\mathcal{O}(\Delta \alpha(t) \Delta \rho)([36,43,44])$.

In the SANC we use replacements (38) at the level of form factors. There are five Born-like form factors: one for $\gamma$ exchange $\tilde{F}_{\gamma}$ that is proportional only to the EW coupling and four for $Z$ exchange: $\tilde{F}_{L L}, \tilde{F}_{L Q}, \tilde{F}_{Q L}, \tilde{F}_{Q Q}$ that are all proportional to the EW coupling and to the factor $\left(s_{W}^{2} c_{W}^{2}\right)^{-1}$ 
from $\chi_{Z}(t)$ Eq. (11). Also each $Q$ form factor has an additional $s_{W}^{2}$, Eq. (14).

We consider the replacement for the form factors in the $\alpha(0)$ and $G_{\mu}$ EW schemes separately.

(i) The $\alpha(0) \mathrm{EW}$ scheme

Let us consider the replacement for the $\tilde{F}_{L L}$ form factor due to all factors listed above,

$$
\begin{aligned}
\alpha(0) & \frac{1}{s_{W}^{2} c_{W}^{2}} \tilde{F}_{L L} \rightarrow \alpha(t) \frac{1}{\bar{s}_{W}^{2} \bar{c}_{W}^{2}} \tilde{F}_{L L} \\
= & \alpha(0) \frac{1}{s_{W}^{2} c_{W}^{2}}\left[1+\Delta \alpha(t)+\Delta \alpha(t)^{2}\right] \\
& \times\left[1-\frac{c_{W}^{2}}{s_{W}^{2}} \Delta \rho+\frac{c_{W}^{4}}{s_{W}^{4}} \Delta \rho^{2}\right]\left[1+\Delta \rho+\Delta \rho^{2}\right] .
\end{aligned}
$$

Removing the common constant from the left and right sides, the leading two-loop form factors read

$$
\begin{aligned}
\tilde{F}_{\gamma} \rightarrow & {\left[1+\Delta \alpha(t)+\Delta \alpha(t)^{2}\right], } \\
\tilde{F}_{L L} \rightarrow & {\left[1+\Delta \alpha(t)+\Delta \alpha(t)^{2}\right]\left[1+\Delta \rho+\Delta \rho^{2}\right] } \\
& \times\left[1-\frac{c_{W}^{2}}{s_{W}^{2}} \Delta \rho+\frac{c_{W}^{4}}{s_{W}^{4}} \Delta \rho^{2}\right], \\
\tilde{F}_{L Q} \rightarrow & {\left[1+\Delta \alpha(t)+\Delta \alpha(t)^{2}\right]\left[1+\Delta \rho+\Delta \rho^{2}\right], } \\
\tilde{F}_{Q L} \rightarrow & {\left[1+\Delta \alpha(t)+\Delta \alpha(t)^{2}\right]\left[1+\Delta \rho+\Delta \rho^{2}\right], } \\
\tilde{F}_{Q Q} \rightarrow & {\left[1+\Delta \alpha(t)+\Delta \alpha(t)^{2}\right]\left[1+\Delta \rho+\Delta \rho^{2}\right] } \\
& \times\left[1+\frac{c_{W}^{2}}{s_{W}^{2}} \Delta \rho\right] .
\end{aligned}
$$

(ii) The $G_{\mu}$ EW scheme

In the $G_{\mu}$ EW scheme the coupling constant already contains additional $s_{W}^{2}$ through the following relation:

$$
\alpha_{G_{\mu}}=\frac{\sqrt{2} G_{\mu} M_{\mathrm{W}}^{2} s_{W}^{2}}{\pi},
$$

and therefore, the replacement for $\alpha_{G_{\mu}}$ could be written as

$$
\alpha_{G_{\mu}} \rightarrow \alpha_{G \mu} \bar{s}_{W}^{2} / s_{W}^{2}=\alpha_{G_{\mu}}\left(1+\frac{c_{W}^{2}}{s_{W}^{2}} \Delta \rho\right) .
$$

Since all form factors for $Z$ exchange have an additional $\left(s_{W}^{2} c_{W}^{2}\right)^{-1}$ factor, one can write

$$
\alpha_{G_{\mu}} \frac{1}{s_{W}^{2} c_{W}^{2}} \rightarrow \alpha_{G_{\mu}} \frac{1}{s_{W}^{2} \bar{c}_{W}^{2}}=\alpha_{G_{\mu}} \frac{1}{s_{W}^{2} c_{W}^{2}}\left(1+\Delta \rho+\Delta \rho^{2}\right) .
$$

Therefore, the form factors at NNLO order read

$$
\begin{aligned}
\tilde{F}_{\gamma} & \rightarrow\left[1+\frac{c_{W}^{2}}{s_{W}^{2}} \Delta \rho\right], \\
\tilde{F}_{L L} & \rightarrow\left[1+\Delta \rho+\Delta \rho^{2}\right], \\
\tilde{F}_{L Q} & \rightarrow\left[1+\Delta \rho+\Delta \rho^{2}\right]\left[1+\frac{c_{W}^{2}}{s_{W}^{2}} \Delta \rho\right], \\
\tilde{F}_{Q L} & \rightarrow\left[1+\Delta \rho+\Delta \rho^{2}\right]\left[1+\frac{c_{W}^{2}}{s_{W}^{2}} \Delta \rho\right], \\
\tilde{F}_{Q Q} & \rightarrow\left[1+\Delta \rho+\Delta \rho^{2}\right]\left[1+\frac{c_{W}^{2}}{s_{W}^{2}} \Delta \rho\right]^{2} .
\end{aligned}
$$

The replacements (39) and (44) should be introduced into helicity amplitudes. After squaring the HAs, all terms higher than $\Delta \rho^{2}, \Delta \rho \Delta \alpha$, and $\Delta \alpha^{2}$ should be omitted. To avoid double counting, one should also remove the leading NLO EW contribution (30) from the terms linear in $\Delta \rho$ : $\Delta \rho \rightarrow\left(\Delta \rho-\left.\Delta \rho^{(1)}\right|^{\alpha(0)}\right)$ and drop the $\Delta \alpha(t)$ contribution.

We have verified analytically that the results obtained in this way agree with the corresponding expressions derived in [45].

\section{NUMERICAL RESULTS AND COMPARISONS}

In this section, we present the numerical results for EW RCs to the $\mu^{+}$and $\mu^{-}$channels of elastic $\mu-e$ scattering at the one-loop level obtained by means of the SANC system.

Numerical results are evaluated for two energy points and the following helicity states of the antimuon/muon $\left(P_{\mu^{ \pm}}\right)$and electron $\left(P_{e^{-}}\right)$beam polarization,

$\left(P_{\mu^{ \pm}}, P_{e^{-}}\right)=(0,0),(-1,-1),(-1,+1),(+1,-1),(+1,+1)$,

in the $\alpha(0)$ and $G_{\mu}$ schemes. Obviously, results for any set of polarizations can be obtained from these cross sections.

A comparison of our results for specific contributions at the tree level with CalcHEP [46] and WHIZARD codes [24] is given.

We used the following set of input parameters taken from the PDG (2020) [47]: 


$$
\begin{aligned}
\alpha^{-1}(0) & =137.035999084, \\
G_{\mu} & =1.1663787 \times 10^{-5} \mathrm{GeV}^{-2} \\
\alpha_{s}\left(M_{\mathrm{Z}}\right) & =0.1179, \\
M_{\mathrm{Z}} & =91.1876 \mathrm{GeV}, \quad \Gamma_{Z}=2.4952 \mathrm{GeV}, \\
M_{\mathrm{W}} & =80.379 \mathrm{GeV} \quad M_{\mathrm{H}}=125.25 \mathrm{GeV}, \\
m_{e} & =0.51099895 \mathrm{MeV}, \\
m_{\mu} & =0.1056583745 \mathrm{GeV}, \\
m_{\tau} & =1.77686 \mathrm{GeV}, \\
m_{u} & =0.062 \mathrm{GeV}, \quad m_{d}=0.083 \mathrm{GeV}, \\
m_{c} & =1.5 \mathrm{GeV}, \quad m_{s}=0.215 \mathrm{GeV}, \\
m_{t} & =172.76 \mathrm{GeV} . \quad m_{b}=4.7 \mathrm{GeV} .
\end{aligned}
$$

We show the results for the polarized Born, hard bremsstrahlung, one-loop cross sections (pb) and relative corrections $(\%)$.

\section{A. Comparisons with other codes}

The polarized Born and hard bremsstrahlung cross sections were cross-checked with the corresponding results of the WHIZARD [24] and CalcHEP [46] programs.

The results are given in the $\alpha(0)$ EW scheme with fixed $100 \%$ polarized initial states for $\sqrt{s}=250 \mathrm{GeV}$ and angular cuts $\left|\cos \vartheta_{\mu}\right| \leq 0.9$ and $\left|\cos \vartheta_{e}\right| \leq 0.9$. For the hard bremsstrahlung cross sections, an additional cut on the photon energy $E_{\gamma} \geq \omega=10^{-4} \sqrt{s} / 2$ is applied.

The results of comparisons are shown in Tables I and II. Very good agreement within 4-5 digits with the above mentioned codes is found.

\section{B. One-loop cross sections and relative corrections}

\section{The case of $\sqrt{s}=250 \mathrm{GeV}$ c.m.s. energy}

In Tables III-IV, we present the values of the Born cross sections (in pb) as well as the relative corrections,

TABLE I. The tuned triple comparison between SANC (the first line), WHIZARD (the second line), and CalcHEP (the third line) results for the hard bremsstrahlung cross section $(\mathrm{pb})$ for $100 \%$ polarized $\mu^{-} e^{-} \rightarrow e^{-} \mu^{-} \gamma$ scattering and for c.m.s. energy $\sqrt{s}=250 \mathrm{GeV}$. For comparison of the real photon emission, we applied an additional cut on the photon energy $E_{\gamma} \geq \omega=10^{-4} \sqrt{s} / 2$. The angular cuts are $\left|\cos \vartheta_{\mu}\right| \leq 0.9$ and $\left|\cos \vartheta_{e}\right| \leq 0.9$.

\begin{tabular}{lccccc}
\hline \hline$P_{\mu^{-}}, P_{e^{-}}$ & 0,0 & $-1,-1$ & $-1,1$ & $1,-1$ & 1,1 \\
\hline $\mathrm{S}$ & $102.42(1)$ & $157.64(1)$ & $56.53(1)$ & $56.52(1)$ & $139.05(1)$ \\
$\mathrm{W}$ & $102.43(1)$ & $157.62(1)$ & $56.53(1)$ & $56.54(1)$ & $139.05(1)$ \\
$\mathrm{C}$ & $102.43(1)$ & $157.63(1)$ & $56.53(1)$ & $56.52(1)$ & $139.06(2)$ \\
\hline \hline
\end{tabular}

TABLE II. The same as in Table I but for $\mu^{+} e^{-} \rightarrow e^{-} \mu^{+} \gamma$ scattering.

\begin{tabular}{lccccc}
\hline \hline$P_{\mu^{+}}, P_{e^{-}}$ & 0,0 & $-1,-1$ & $-1,1$ & $1,-1$ & 1,1 \\
\hline $\mathrm{S}$ & $91.63(1)$ & $77.27(1)$ & $99.88(1)$ & $112.12(1)$ & $77.28(1)$ \\
$\mathrm{W}$ & $91.63(1)$ & $77.28(1)$ & $99.90(1)$ & $112.12(1)$ & $77.28(1)$ \\
$\mathrm{C}$ & $91.63(1)$ & $77.27(1)$ & $99.89(1)$ & $112.11(1)$ & $77.27(1)$ \\
\hline \hline
\end{tabular}

$$
\delta^{\mathrm{Y}}=\frac{\sigma^{\mathrm{Y}}}{\sigma^{\mathrm{Born}}}, \%
$$

for reactions (1-2) for the c.m.s. energy $\sqrt{s}=250 \mathrm{GeV}$ with angular cuts $\left|\cos \vartheta_{\mu}\right| \leq 0.9$ and $\left|\cos \vartheta_{e}\right| \leq 0.9$. We consider the unpolarized and fully (100\%) polarized initial beams (44) in the $\alpha(0)$ and $G_{\mu}$ EW schemes.

In the tables, particular contributions of the NLO EW relative corrections as well as the leading h.o. corrections are shown. We split the complete one-loop contribution into two gauge-invariant subsets of the diagrams $\delta^{\mathrm{QED}}$ and $\delta^{\text {weak }}$, where $\delta^{\text {weak }}$ includes vacuum polarization (vp) as well as pure weak-interaction contributions; $\delta^{\text {weak-vp }}=\delta^{\text {weak }}-\delta^{\mathrm{vp}}$.

It is convenient to discuss both tables in the same manner. A comparison of the cross sections with different values of polarization has demonstrated the significance of polarization effects. Namely, for the $\mu^{+}$channel the cross section $\sigma_{+-}$is about 1.5 times larger than $\sigma_{--,++}$and about 1.2 times larger than the unpolarized cross section. As for the $\mu^{-}$channel, the cross section $\sigma_{--}$is about 2.5 times larger than $\sigma_{-+,+-}$and about 1.5 times larger than the unpolarized cross section.

The QED part of the relative RCs for the unpolarized and fully polarized cases in the $\alpha(0)$ and $G_{\mu}$ EW schemes is negative and has a maximum of about $3 \%$ in the $\mu^{+}$ channel for $\delta_{+-,-+}^{\text {QED }}$ (the same situation is in the $\mu^{-}$channel;

TABLE III. Integrated Born cross sections and relative corrections for $\mu^{+} e^{-} \rightarrow e^{-} \mu^{+}(\gamma)$ scattering for the c.m.s. energy $\sqrt{s}=$ $250 \mathrm{GeV}$ and the set (44) of polarization degrees of the initial particles in the $\alpha(0)$ and $G_{\mu} \mathrm{EW}$ schemes.

\begin{tabular}{llllll}
\hline \hline$P_{\mu^{+}}, P_{e^{-}}$ & \multicolumn{1}{c}{0,0} & $-1,-1$ & $-1,+1$ & $+1,-1$ & $+1,+1$ \\
\hline \multicolumn{5}{c}{$\alpha(0)$ EW } & scheme \\
$\sigma^{\text {Born }}, \mathrm{pb}$ & $66.487(1)$ & $55.333(1)$ & $73.186(1)$ & $82.097(1)$ & $55.333(1)$ \\
$\delta^{\mathrm{QED}}, \%$ & $-1.936(1)$ & $-0.481(1)$ & $-2.933(1)$ & $-3.013(1)$ & $-0.482(1)$ \\
$\delta^{\mathrm{VP}}, \%$ & $11.466(1)$ & $13.729(2)$ & $10.151(1)$ & $9.586(1)$ & $13.729(2)$ \\
$\delta^{\text {weak-VP } \%}$ & $-0.396(1)$ & $-1.758(1)$ & $2.297(1)$ & $-0.962(1)$ & $-1.758(1)$ \\
$\delta^{\mathrm{ho}}, \%$ & $1.032(1)$ & $0.929(1)$ & $0.895(1)$ & $1.295(1)$ & $0.929(1)$ \\
& & & & \\
$\sigma^{\mathrm{Born}}, \mathrm{pb}$ & $71.458(1)$ & $59.470(1)$ & $78.658(1)$ & $88.234(1)$ & $59.470(1)$ \\
$\delta^{\mathrm{QED}}, \%$ & $-1.935(2)$ & $-0.481(2)$ & $-2.930(2)$ & $-3.007(2)$ & $-0.482(2)$ \\
$\delta^{\mathrm{VP}}, \%$ & $5.568(1)$ & $6.705(1)$ & $4.899(1)$ & $4.630(1)$ & $6.705(2)$ \\
$\delta^{\text {weak-VP }}, \%$ & $-0.391(1)$ & $-0.626(1)$ & $1.656(1)$ & $-1.891(1)$ & $-0.626(1)$ \\
$\delta^{\mathrm{ho}}, \%$ & $-0.456(1)$ & $-0.512(1)$ & $-0.520(1)$ & $-0.322(1)$ & $-0.512(1)$ \\
\hline \hline
\end{tabular}


TABLE IV. The same as in Table III but for $\mu^{-} e^{-} \rightarrow e^{-} \mu^{-}(\gamma)$ scattering.

\begin{tabular}{|c|c|c|c|c|c|}
\hline$P_{\mu^{+}}, P_{e^{-}}$ & 0,0 & $-1,-1$ & $-1,+1$ & $+1,-1$ & $+1,+1$ \\
\hline \multicolumn{6}{|c|}{$\alpha(0)$ EW scheme } \\
\hline$\sigma^{\text {Born }}, \mathrm{pb}$ & $75.231(1)$ & $115.076(1)$ & $42.157(1)$ & $42.157(1)$ & $101.538(1)$ \\
\hline$\delta^{\mathrm{QED}}, \%$ & $-2.085(1)$ & $-1.912(1)$ & $-2.682(1)$ & $-2.683(1)$ & $-1.781(1)$ \\
\hline$\delta^{\mathrm{VP}}, \%$ & $10.849(1)$ & $9.602(1)$ & $13.305(1)$ & $13.305(1)$ & $10.220(1)$ \\
\hline$\delta^{\text {weak-VP }}, \%$ & $-0.161(1)$ & $-1.476(1)$ & $-1.540(1)$ & $-1.540(1)$ & $2.474(1)$ \\
\hline$\delta^{\text {ho }}, \%$ & $1.089(1)$ & $1.365(1)$ & $0.907(1)$ & $0.907(1)$ & $0.926(1)$ \\
\hline \multicolumn{6}{|c|}{$G_{\mu}$ EW scheme } \\
\hline$\sigma^{\text {Born }}, \mathrm{pb}$ & $80.855(1)$ & $123.679(1)$ & $45.309(1)$ & $45.309(1)$ & $109.128(1)$ \\
\hline$\delta^{\mathrm{QED}}, \%$ & $-2.082(2)$ & $-1.911(1)$ & $-2.685(2)$ & $-2.685(2)$ & $-1.780(1)$ \\
\hline$\delta^{\mathrm{VP}}, \%$ & $5.295(1)$ & $4.729(1)$ & $6.393(1)$ & $6.393(1)$ & $5.027(1)$ \\
\hline$\delta^{\text {weak-VP }}, \%$ & $-0.501(1)$ & $-2.495(1)$ & $-0.519(1)$ & $-0.519(1)$ & $1.775(1)$ \\
\hline$\delta^{\mathrm{ho}}, \%$ & $-0.436(1)$ & $-0.306(1)$ & $-0.511(1)$ & $-0.511(1)$ & $-0.522(1)$ \\
\hline
\end{tabular}

i.e., the maximum is about $2.7 \%$ for the same polarization values), and has a minimum of about $-0.5 \%$ in the $\mu^{+}$ channel for $\delta_{--,++}^{\mathrm{QED}}$ [about (1.8-1.9)\% in the $\mu^{-}$channel].

The main contribution to the weak correction is due to the vacuum polarization $\delta^{\mathrm{vp}}$, which is positive and gives about $10 \%-13 \%$ in the $\alpha(0)$ EW scheme (5\%-7\% in the $G_{\mu}$ scheme) for both $\mu^{+}$and $\mu^{-}$channels.

The rest of the weak correction $\delta^{\text {weak-vp }}$ is also negative, and it can reach up to $1.5 \%-2 \%$ and even can change the sign.

The leading higher-order corrections are positive and equal to about $1 \%$ in the $\alpha(0)$ EW scheme while in the $G_{\mu}$ one they are negative and equal to about $0.5 \%$.

All weak and h.o. corrections strongly depend on the choice of the EW scheme, and the total weak corrections in $G_{\mu}$ scheme are smaller by about $5 \%-6 \%$ than in the $\alpha(0)$ one.

In Tables V and VI, the integrated cross sections for the weak and leading higher-order corrections are shown for both $\mu^{+}$and $\mu^{-}$channels. The results are calculated in the $\alpha(0)$ and $G_{\mu}$ schemes, their difference,

TABLE V. Weak and higher-order corrected cross sections for $\mu^{+} e^{-} \rightarrow e^{-} \mu^{+}(\gamma)$ scattering for the c.m.s. energy $\sqrt{s}=250 \mathrm{GeV}$ and the set (44) of polarization degrees of the initial particles in the $\alpha(0)$ and $G_{\mu}$ EW schemes.

\begin{tabular}{llllll}
\hline \hline$P_{\mu^{+}}, P_{e^{-}}$ & \multicolumn{1}{c}{0,0} & \multicolumn{1}{c}{$-1,-1$} & \multicolumn{1}{c}{$-1,+1$} & $+1,-1$ & $+1,+1$ \\
\hline$\sigma_{\alpha(0)}^{\text {weak }}, \mathrm{pb}$ & $73.846(1)$ & $61.956(1)$ & $82.295(1)$ & $89.175(1)$ & $61.956(1)$ \\
$\sigma_{G_{\mu}}^{\text {weak }}, \mathrm{pb}$ & $75.156(1)$ & $63.084(1)$ & $83.812(1)$ & $90.642(1)$ & $63.084(1)$ \\
$\delta_{G_{\mu} / \alpha(0)}^{\text {weak }}, \%$ & 1.77 & 1.82 & 1.84 & 1.65 & 1.82 \\
$\sigma_{\alpha(0)}^{\text {weak+ho }}, \mathrm{pb}$ & $74.533(1)$ & $62.471(1)$ & $82.951(1)$ & $90.240(1)$ & $62.471(1)$ \\
$\sigma_{G_{\mu}}^{\text {weak }+ \text { ho }}, \mathrm{pb}$ & $74.830(1)$ & $62.779(1)$ & $83.405(1)$ & $90.359(1)$ & $62.779(1)$ \\
$\delta_{G_{\mu} / \alpha(0)}^{\text {weak }+ \text { ho }}, \%$ & 0.40 & 0.50 & 0.55 & 0.13 & 0.50 \\
\hline \hline
\end{tabular}

TABLE VI. The same as in Table V but for $\mu^{-} e^{-} \rightarrow e^{-} \mu^{-}(\gamma)$ scattering.

\begin{tabular}{|c|c|c|c|c|c|}
\hline$P_{\mu^{+}}, P_{e^{-}}$ & 0,0 & $-1,-1$ & $-1,+1$ & $+1,-1$ & $+1,+1$ \\
\hline$\sigma_{\alpha(0)}^{\mathrm{weak}}, \mathrm{pb}$ & $82.272(1)$ & $124.427(1)$ & $47.117(1)$ & $47.117(1)$ & $114.427(1)$ \\
\hline$\sigma_{G_{\mu}}^{\mathrm{weak}}, \mathrm{pb}$ & $84.732(1)$ & $126.441(1)$ & $47.969(1)$ & $47.969(1)$ & $116.551(1)$ \\
\hline$\delta_{G_{\mu} / \alpha(0)}^{\text {weak }}, \%$ & 2.99 & 1.62 & 1.81 & 1.81 & 1.86 \\
\hline$\sigma_{\alpha(0)}^{\mathrm{weak}+\mathrm{ho}}, \mathrm{pb}$ & 84.091(1) & $125.999(1)$ & $47.499(1)$ & $47.499(1)$ & $115.368(1)$ \\
\hline$\sigma_{G_{\mu}}^{\text {weak }+ \text { ho }}, \mathrm{pb}$ & $84.379(1)$ & $126.062(1)$ & $47.738(1)$ & $47.738(1)$ & $115.981(1)$ \\
\hline$\delta_{G_{\mu} / \alpha(0)}^{\text {weak+ho }}, \%$ & 0.34 & 0.05 & 0.05 & 0.50 & 0.53 \\
\hline
\end{tabular}

is also shown. Ratio (45) shows the stabilization of the results and can be considered as an estimation of the theoretical uncertainty of the weak and h.o. contributions. As it is well known, the difference between two EW schemes in the LO is just the ratio of the EW couplings and gives about $\delta_{G_{\mu} / \alpha(0)}^{\mathrm{LO}}=7 \%$. As it is seen from the tables, the weak contribution reduces the difference almost by one half to about $\delta_{G_{\mu} / \alpha(0)}^{\mathrm{weak}}=2 \%-3 \%$. Even more, the sum of the weak and h.o. contributions reduces the difference almost by one half or one third to about $\delta_{G_{\mu} / \alpha(0)}^{\mathrm{weak}+\mathrm{h} . \mathrm{o}}=$ $0.05 \%-0.5 \%$ depending on the value of polarization degrees and the reaction channel. We consider $0.5 \%$ as a rather big difference which is caused by using the Mandelstam variable $t$ as a scale of the $\Delta \alpha(t)$ (33) quantity in calculations of the h.o. contributions within the $\alpha(0)$ EW scheme.

Figures 1-4 show the differential cross sections for LO and NLO EW and relative corrections as functions of the $\cos \theta_{e}$ and $\cos \theta_{\mu}^{ \pm}$, respectively. The maxima for the LO and



FIG. 1. The LO and NLO EW cross sections (upper panel) and relative corrections (lower panel) of the $\mu^{+} e^{-} \rightarrow e^{-} \mu^{+}(\gamma)$ process for the c.m.s. energy $\sqrt{s}=250 \mathrm{GeV}$ as a function of $\cos \theta_{e}$. 


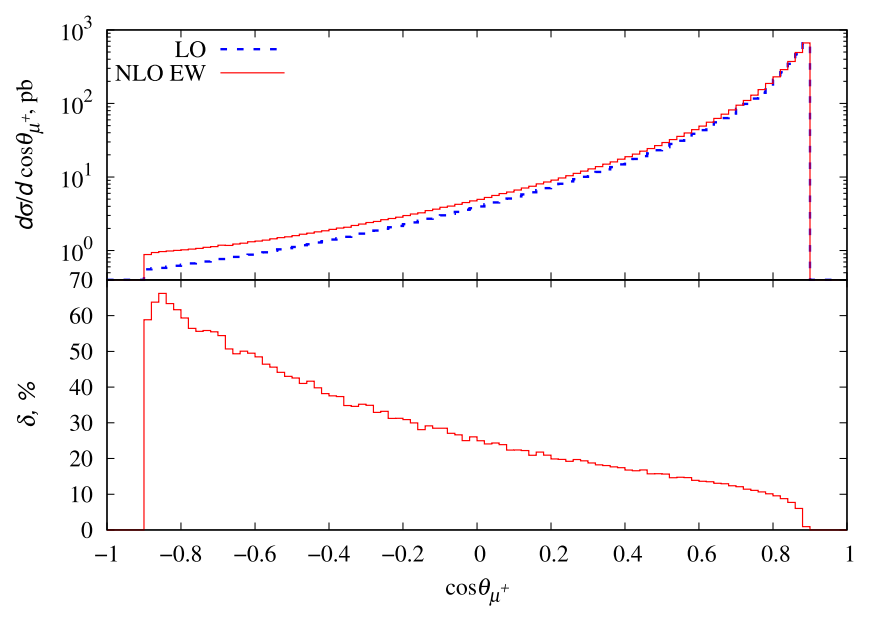

FIG. 2. The same as in Fig. 1 but for $\cos \theta_{\mu^{+}}$.

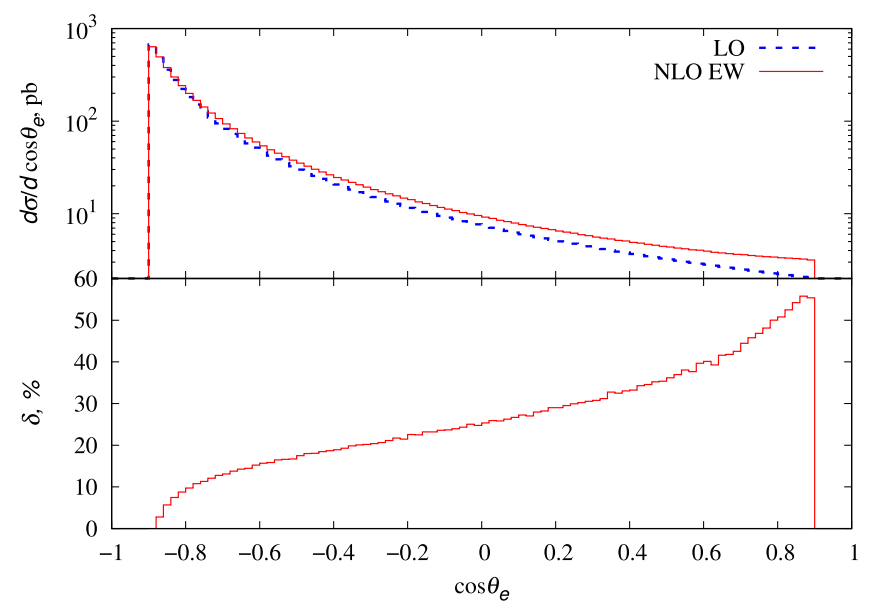

FIG. 3. The LO and NLO EW cross sections (upper panel) and relative corrections (lower panel) of the $\mu^{-} e^{-} \rightarrow e^{-} \mu^{-}(\gamma)$ process for the c.m.s. energy $\sqrt{s}=250 \mathrm{GeV}$ as a function of $\cos \theta_{e}$.

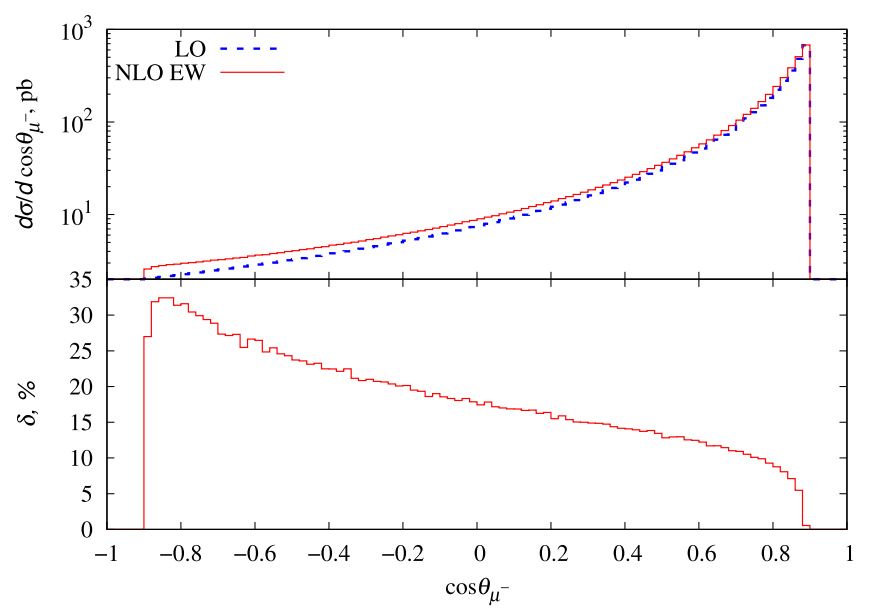

FIG. 4. The same as in Fig. 3 but for $\cos \theta_{\mu^{-}}$.
NLO EW cross section are at the $t \rightarrow 0\left(\cos \theta_{e} \simeq-1\right.$ of the final electron and $\cos \theta_{\mu}^{ \pm} \simeq 1$ of the final (anti)muon). At the same time, the relative corrections have maxima at opposite values of angles where real photon contribution dominates [48].

\section{The case of the laboratory system energy $E_{\mu}=150 \mathrm{GeV}, E_{e}=m_{e}$}

In order to validate the code, we have calculated NLO EW and h.o. corrections for the setup of the MUonE experiment proposed in [10]. Namely, we have used setup 1: $\quad E_{\mu}=150 \mathrm{GeV}, \quad E_{e}=m_{e} \quad(\sqrt{s} \simeq 0.405541 \mathrm{GeV})$, $\theta_{e}, \theta_{\mu} \leq 100 \mathrm{mrad}, E_{e} \geq 0.2 \mathrm{GeV}$.

The results are shown in Tables VII and VIII for both muon channels and for the set of polarization degrees (44) of the initial particles in the $\alpha(0) \mathrm{EW}$ scheme. In the tables, we show the integrated Born cross sections and the relative corrections for the QED part, the vacuum polarization part of the weak contribution, and the part of h.o. corrections proportional to $\Delta \alpha(t)^{2}$. The rest of the weak contribution $\delta^{\text {weak-vp }}$ which is about $10^{-4} \%$ and the rest of h.o. corrections [not proportional to $\Delta \alpha(t)^{2}$ ] is about $10^{-8 \%}$, they are omitted in the tables.

Tables VII and VIII show that the effect of the initial particle polarization changes the third digits in $\delta^{\mathrm{QED}}$ and $\delta^{\mathrm{vp}}$. The integrated cross section $\sigma_{--,++}$is larger than $\sigma_{\text {unp }}$ by about $0.8 \%$ for both $\mu^{+}$and $\mu^{-}$channels. The h.o. contribution from $\Delta \alpha(t)^{2}$ is about $6 \times 10^{-3} \%$.

Figures 5 and 6 show the differential cross for LO and NLO QED and relative corrections as functions of the variables $t_{e e}=\left(p_{2}-p_{3}\right)^{2}$ and $t_{\mu \mu}=\left(p_{1}-p_{4}\right)^{2}$, respectively. In Figs. 7 and 8 , the distributions on the final

TABLE VII. Integrated Born cross section and relative corrections for $\mu^{+} e^{-} \rightarrow e^{-} \mu^{+}(\gamma)$ scattering for the laboratory system energy $E_{\mu}=150 \mathrm{GeV}$ and the set (44) of the initial particle polarization degrees in the $\alpha(0)$ EW scheme.

\begin{tabular}{llllll}
\hline \hline$P_{\mu^{+}}, P_{e^{-}}$ & \multicolumn{1}{c}{0,0} & \multicolumn{1}{c}{$-1,-1$} & $-1,+1$ & $+1,-1$ & $+1,+1$ \\
\hline$\sigma^{\mathrm{Born}}, \mu \mathrm{b}$ & $1265.1(1)$ & $1275.3(1)$ & $1254.8(1)$ & $1254.8(1)$ & $1275.3(1)$ \\
$\delta^{\mathrm{QED}}, \%$ & $4.762(1)$ & $4.766(1)$ & $4.757(1)$ & $4.759(1)$ & $4.765(1)$ \\
$\delta^{\mathrm{VP}}, \%$ & $0.940(1)$ & $0.943(1)$ & $0.936(1)$ & $0.936(1)$ & $0.943(1)$ \\
$\delta^{\mathrm{ho}}\left(\Delta \alpha^{2}\right), \%$ & $0.006(1)$ & $0.006(1)$ & $0.006(1)$ & $0.006(1)$ & $0.006(1)$ \\
\hline \hline
\end{tabular}

TABLE VIII. The same as in Table VII but for $\mu^{-} e^{-} \rightarrow$ $e^{-} \mu^{-}(\gamma)$ scattering.

\begin{tabular}{llllll}
\hline \hline$P_{\mu^{-}}, P_{e^{-}}$ & \multicolumn{1}{c}{0,0} & $-1,-1$ & $-1,+1$ & $+1,-1$ & $+1,+1$ \\
\hline$\sigma^{\mathrm{Born}}, \mu \mathrm{b}$ & $1265.1(1)$ & $1275.3(1)$ & $1254.8(1)$ & $1254.8(1)$ & $1275.3(1)$ \\
$\delta^{\mathrm{QED}}, \%$ & $4.624(1)$ & $4.608(1)$ & $4.640(1)$ & $4.639(1)$ & $4.608(1)$ \\
$\delta^{\mathrm{VP}}, \%$ & $0.940(1)$ & $0.940(1)$ & $0.940(1)$ & $0.940(1)$ & $0.940(1)$ \\
$\delta^{\mathrm{ho}}\left(\Delta \alpha^{2}\right), \%$ & $0.006(1)$ & $0.006(1)$ & $0.006(1)$ & $0.006(1)$ & $0.006(1)$ \\
\hline \hline
\end{tabular}




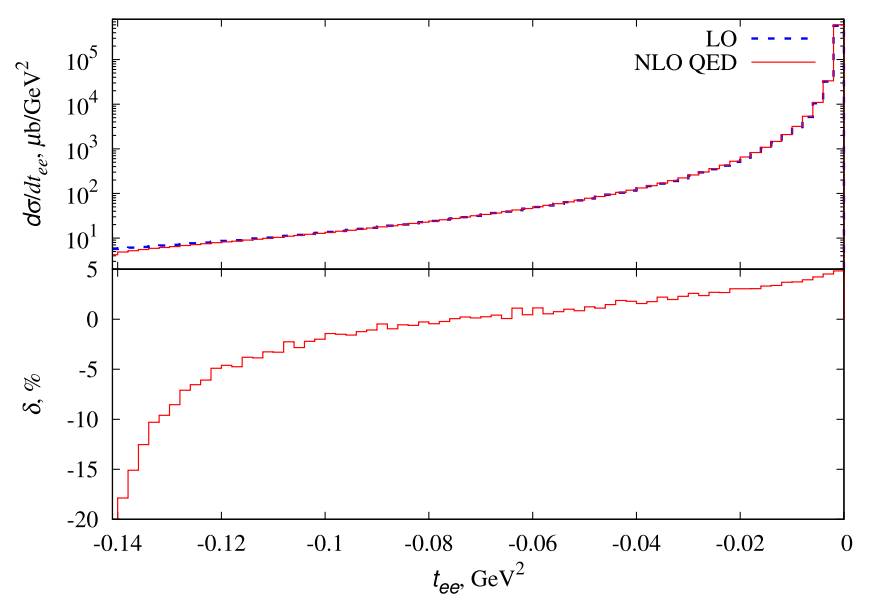

FIG. 5. The LO and NLO QED cross sections (upper panel) and relative corrections (lower panel) of the $\mu^{+} e^{-} \rightarrow e^{-} \mu^{+}(\gamma)$ process for the laboratory system energy $E_{\mu}=150 \mathrm{GeV}$ as a function of $t_{e e}=\left(p_{2}-p_{3}\right)^{2}$.

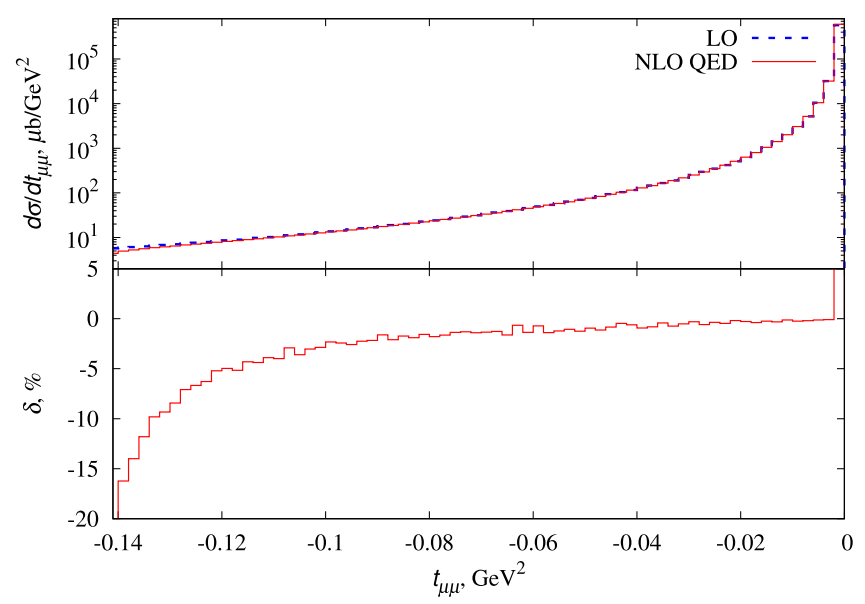

FIG. 6. The same as in Fig. 5 but for $t_{\mu \mu}=\left(p_{1}-p_{4}\right)^{2}$.

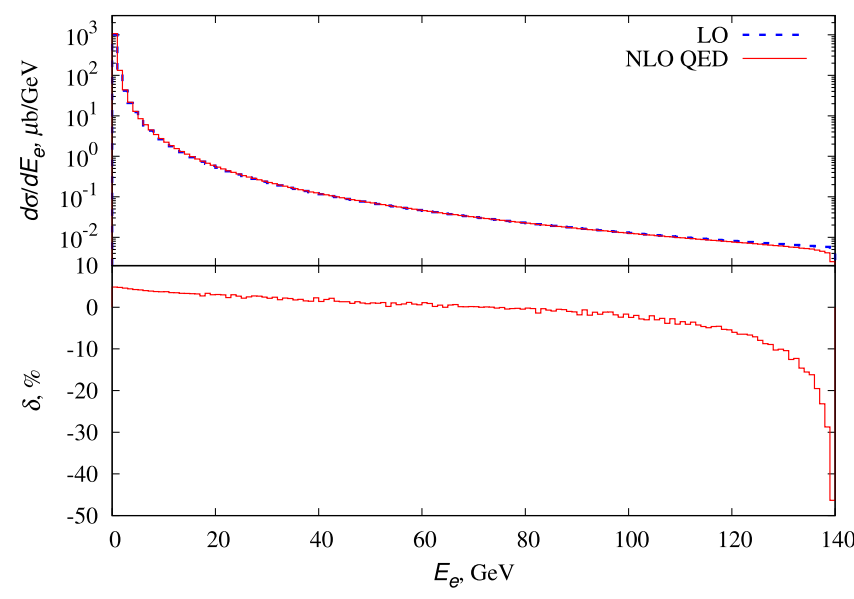

FIG. 7. The same as in Fig. 5 but for electron energy $E_{e}$.

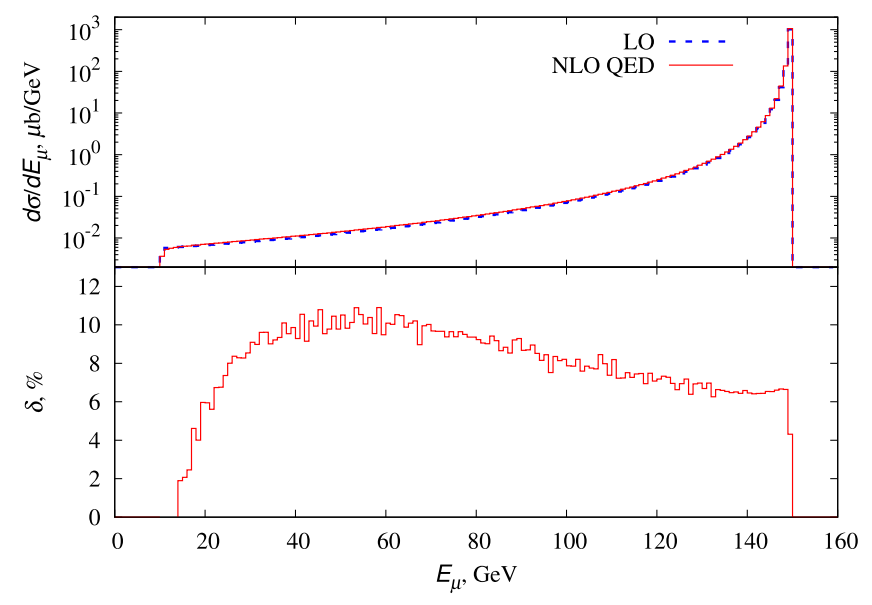

FIG. 8. The same as in Fig. 5 but for muon energy $E_{\mu}$.

electron $E_{e}$ and muon $E_{\mu}$ energies are presented. All distributions look very similar to those given in [10].

\section{CONCLUSIONS}

In this paper, we have described the implementation of complete one-loop EW corrections to elastic $\mu-e$ scattering within the SANC system framework. The relevant contributions to the cross section are calculated analytically using the helicity amplitude approach, which allows us to take into account any polarization. The helicity amplitudes were used for Born and virtual parts as well as for the soft and hard photon bremsstrahlung, taking into account the masses of the initial and final fermions.

The numerical results are also presented. The effects of initial beam polarization were analyzed for fully polarized states. Two energy points were considered: the c.m.s. energy $\sqrt{s}=250 \mathrm{GeV}$ and the laboratory system energy $E_{\mu}=150 \mathrm{GeV}$.

The calculated polarized cross sections at the tree level for Born and hard photon bremsstrahlung were thoroughly compared with the results of CalcHEP and WHIZARD, and very good agreement with the above-mentioned codes was observed.

It was found that cross sections strongly depend on polarization degrees of initial beams. As a result, the polarization effects are significant and give increase in the cross sections at definite polarization degrees.

The complete one-loop and leading higher-order corrections were analyzed. Higher-order terms were introduced by using the parameters $\Delta \alpha$ and $\Delta \rho$.

For the c.m.s. energy $\sqrt{s}=250 \mathrm{GeV}$ calculations in $\alpha(0)$, and $G_{\mu}$ EW schemes were performed. The sum of weak and higher-order contributions reduces the difference between the results in two EW schemes to about $0.05 \%-0.5 \%$ depending on the polarization values and the reaction channel. This part of our study can be considered as a preliminary step in preparation of a 
contribution to the physical program of the proposed $\mu$ TRISTAN experiment [5].

For the laboratory system energy $E_{\mu}=150 \mathrm{GeV}$, we present cross sections and distributions calculated with up to date input parameters.

The forthcoming part of our work on this process will be devoted to calculation of leading and next-to-leading large logarithmic corrections in higher orders $\alpha^{n} L^{n}$ and $\alpha^{n} L^{n-1}$, where $L=\ln \left(Q^{2} / m_{e}^{2}\right)$ and $n \geq 3$.

\section{ACKNOWLEDGMENTS}

This research was funded by RFBR, Project No. 2002-00441.
[1] T. V. Kukhto, N. M. Shumeiko, and S. I. Timoshin, J. Phys. G 13, 725 (1987).

[2] N. Kaiser, J. Phys. G 37, 115005 (2010).

[3] D. Adams et al. (Spin Muon (SMC) Collaboration), Phys. Lett. B 396, 338 (1997).

[4] D. Yu. Bardin and L. Kalinovskaya, arXiv:hep-ph/9712310.

[5] Y. Hamada, R. Kitano, R. Matsudo, H. Takaura, and M. Yoshida, arXiv:2201.06664.

[6] G. Abbiendi et al., Eur. Phys. J. C 77, 139 (2017).

[7] G. Abbiendi, Letter of intent: The MUonE project, CERN Report No. CERN-SPSC-2019-026, 2019, https://cds.cern .ch/record/2677471.

[8] A. Masiero, P. Paradisi, and M. Passera, Phys. Rev. D 102, 075013 (2020).

[9] P. S. B. Dev, W. Rodejohann, X.-J. Xu, and Y. Zhang, J. High Energy Phys. 05 (2020) 053.

[10] M. Alacevich, C. M. Carloni Calame, M. Chiesa, G. Montagna, O. Nicrosini, and F. Piccinini, J. High Energy Phys. 02 (2019) 155.

[11] C. M. Carloni Calame, M. Chiesa, G. Montagna, O. Nicrosini, and F. Piccinini, EPJ Web Conf. 212, 05002 (2019).

[12] C. M. Carloni Calame, M. Chiesa, S. M. Hasan, G. Montagna, O. Nicrosini, and F. Piccinini, J. High Energy Phys. 11 (2020) 028.

[13] E. Budassi, C. M. Carloni Calame, M. Chiesa, C. L. Del Pio, S. M. Hasan, G. Montagna, O. Nicrosini, and F. Piccinini, J. High Energy Phys. 11 (2021) 098.

[14] P. Banerjee, T. Engel, A. Signer, and Y. Ulrich, SciPost Phys. 9, 027 (2020).

[15] Y. Ulrich, McMule-QED Corrections for Low-Energy Experiments, other thesis, Universität Zürich, 2020, arXiv: 2008.09383.

[16] M. Fael and M. Passera, Phys. Rev. Lett. 122, 192001 (2019).

[17] E. Balzani, Leptonic QED contributions to muon-electron scattering at NNLO, Master's thesis, Padua University, 2020, https://inspirehep.net/files/aeb955823dc2c19c3874a 9db109221dd.

[18] P. Banerjee et al., Eur. Phys. J. C 80, 591 (2020).

[19] A. Andonov, A. Arbuzov, D. Bardin, S. Bondarenko, P. Christova, L. Kalinovskaya, G. Nanava, and W. von Schlippe, Comput. Phys. Commun. 174, 481 (2006); 177, 623(E) (2007).

[20] D. Yu. Bardin et al., Phys. Part. Nucl. 50, 395 (2019).
[21] D. Bardin, Y. Dydyshka, L. Kalinovskaya, L. Rumyantsev, A. Arbuzov, R. Sadykov, and S. Bondarenko, Phys. Rev. D 98, 013001 (2018).

[22] S. Bondarenko, Y. Dydyshka, L. Kalinovskaya, R. Sadykov, and V. Yermolchyk, Phys. Rev. D 102, 033004 (2020).

[23] A. Andonov, D. Bardin, S. Bondarenko, P. Christova, L. Kalinovskaya, and G. Nanava, Fiz. Elem. Chastits At. Yadra 34, 1125 (2003), http://www1.jinr.ru/Archive/ Pepan/2003-v34/v-34-5/pdf_obzory/v34p5_02.pdf; [Phys. Part. Nucl. 34, 577 (2003)].

[24] W. Kilian, T. Ohl, and J. Reuter, Eur. Phys. J. C 71, 1742 (2011).

[25] G.-P. Passarino and M. J. G. Veltman, Nucl. Phys. B160, 151 (1979).

[26] G. Moortgat-Pick et al., Phys. Rep. 460, 131 (2008).

[27] A. Arbey et al., Eur. Phys. J. C 75, 371 (2015).

[28] S. D. Badger, E. W. N. Glover, V. V. Khoze, and P. Svrcek, J. High Energy Phys. 07 (2005) 025.

[29] S. D. Badger, E. W. N. Glover, and V. V. Khoze, J. High Energy Phys. 01 (2006) 066.

[30] D. Maître and P. Mastrolia, Comput. Phys. Commun. 179, 501 (2008).

[31] S. Bondarenko, Y. Dydyshka, L. Kalinovskaya, L. Rumyantsev, R. Sadykov, and V. Yermolchyk, Phys. Rev. D 100, 073002 (2019).

[32] C. Schwinn and S. Weinzierl, J. High Energy Phys. 04 (2007) 072.

[33] E. P. Wigner, Rev. Mod. Phys. 29, 255 (1957).

[34] E. P. Wigner, Phys. Today 17, No. 3, 34 (1964).

[35] M. Veltman, Nucl. Phys. B123, 89 (1977).

[36] J. Fleischer, O. V. Tarasov, and F. Jegerlehner, Phys. Lett. B 319, 249 (1993).

[37] J. Fleischer, O. V. Tarasov, and F. Jegerlehner, Phys. Rev. D 51, 3820 (1995).

[38] D. Yu. Bardin, W. Hollik, and G. Passarino, Reports of the Working Group on Precision Calculations for the $Z$ Resonance, CERN Yellow Reports: Monographs (CERN, Geneva, 1995), 10.5170/CERN-1995-003.

[39] D. Yu. Bardin, P. Christova, M. Jack, L. Kalinovskaya, A. Olchevski, S. Riemann, and T. Riemann, Comput. Phys. Commun. 133, 229 (2001).

[40] R. Barbieri, M. Beccaria, P. Ciafaloni, G. Curci, and A. Vicere, Phys. Lett. B 288, 95 (1992).

[41] A. Djouadi and C. Verzegnassi, Phys. Lett. B 195, 265 (1987). 
[42] A. Djouadi, Nuovo Cimento A 100, 357 (1988).

[43] M. Consoli, W. Hollik, and F. Jegerlehner, Phys. Lett. B 227, 167 (1989).

[44] M. Consoli, W. Hollik, and F. Jegerlehner, Electroweak radiative corrections for $Z$ physics, CERN Report No. CERN-TH-552789, 1989, 10.5170/CERN-1989-008-V-1.7.

[45] S. Dittmaier and M. Huber, J. High Energy Phys. 01 (2010) 060.
[46] A. Belyaev, N. D. Christensen, and A. Pukhov, Comput. Phys. Commun. 184, 1729 (2013).

[47] P. A. Zyla et al. (Particle Data Group), Prog. Theor. Exp. Phys. 2020, 083 C01 (2020).

[48] It should be noted that in [21] all figures are plotted as a function of the $\cos \left(\widehat{\vec{p}}_{2} \vec{p}_{3}\right)$. 